\title{
Forced fluid dynamics from blackfolds in general supergravity backgrounds
}

\author{
Jay Armas, ${ }^{a}$ Jakob Gath, ${ }^{b}$ Vasilis Niarchos, ${ }^{c}$ Niels A. Obers ${ }^{d}$ and \\ Andreas Vigand Pedersen ${ }^{d}$ \\ ${ }^{a}$ Physique Théorique et Mathématique, \\ Université Libre de Bruxelles and International Solvay Institutes, \\ ULB-Campus Plaine CP231, B-1050 Brussels, Belgium \\ ${ }^{b}$ Centre de Physique Théorique, École Polytechnique, \\ CNRS UMR 7644, Université Paris-Saclay, \\ F-91128 Palaiseau, France \\ ${ }^{c}$ Crete Center for Theoretical Physics, Institute of Theoretical and Computational Physics, \\ Crete Center for Quantum Complexity and Nanotechnology, \\ Department of Physics, University of Crete, \\ Heraklion, 71303, Greece \\ ${ }^{d}$ The Niels Bohr Institute, Copenhagen University, \\ Blegdamsvej 17, DK-2100 Copenhagen Ø, Denmark \\ E-mail: jarmas@ulb.ac.be, jakob.gath@polytechnique.edu, \\ niarchos@physics.uoc.gr, obers@nbi.ku.dk, andreasvigand@gmail.com
}

ABSTRACT: We present a general treatment of the leading order dynamics of the collective modes of charged dilatonic $p$-brane solutions of (super)gravity theories in arbitrary backgrounds. To this end we employ the general strategy of the blackfold approach which is based on a long-wavelength derivative expansion around an exact or approximate solution of the (super)gravity equations of motion. The resulting collective mode equations are formulated as forced hydrodynamic equations on dynamically embedded hypersurfaces. We derive them in full generality (including all possible asymptotic fluxes and dilaton profiles) in a far-zone analysis of the (super)gravity equations and in representative examples in a near-zone analysis. An independent treatment based on the study of external couplings in hydrostatic partition functions is also presented. Special emphasis is given to the forced collective mode equations that arise in type IIA/B and eleven-dimensional supergravities, where besides the standard Lorentz force couplings our analysis reveals additional couplings to the background, including terms that arise from Chern-Simons interactions. We also present a general overview of the blackfold approach and some of the key conceptual issues that arise when applied to arbitrary backgrounds.

KEYwords: Black Holes, Black Holes in String Theory, D-branes, p-branes

ARXIV EPRINT: 1606.09644 


\section{Contents}

1 Introduction 1

2 Far-zone analysis $\quad 5$

2.1 Einstein-dilaton theory with a $(q+1)$-form gauge field 7

2.2 Type IIA/B supergravity 9

2.3 Eleven-dimensional supergravity 11

3 Near-zone analysis $\quad \mathbf{1 2}$

$\begin{array}{lll}3.1 & \text { Constraint equations for charged black branes } & 13\end{array}$

$\begin{array}{ll}3.2 \text { Constraint equations for the } \mathrm{D} p \text {-F1 bound state } & 18\end{array}$

4 External couplings from hydrostatic partition functions 21

4.1 General considerations on partition functions 22

4.2 External couplings for black branes carrying Maxwell charge 24

4.3 External couplings for black branes carrying $q=p$-brane charge 27

5 Conclusions 31

5.1 Comments on the long-wavelength expansions of the blackfold approach 31

5.2 Open problems 36

$\begin{array}{ll}\text { A Notation and conventions } & 39\end{array}$

B Explicit form of type IIA/B probe brane equations $\quad 39$

C Effective currents, charges and constraint equations $\quad 41$

C.1 Black branes carrying $q=p$-brane charge $\quad 41$

C.2 Black branes carrying Maxwell charge 42

C.3 D $p$-F1 bound state 42

C.4 D $p$-F1 constraint equations 43

D Entropy current analysis with a dilaton forcing function 43

\section{Introduction}

Hydrodynamics has proven to be a powerful universal description of the low energy effective dynamics of interacting quantum systems at finite temperature, valid in the regime where fluctuations have sufficiently long wavelength. Modern developments have revealed a close connection between hydrodynamics and gravity, in particular black holes, starting with the pioneering work in AdS/CFT [1, 2] and the discovery of fluid/gravity duality [3, 4] (see [5] for a review). In the latter case the fluid lives on the AdS boundary, but more general fluid dynamical descriptions of black brane dynamics in diverse asymptotic spacetimes (including 
flat space) have also been found in the context of the blackfold approach [6-8]. The relation between AdS fluid/gravity and flat space blackfolds in the context of D3-branes has been considered in [9].

In hydrodynamics the dynamical equations of a system are captured by the combined set of a few conservation equations (typically, the conservation equations of the stressenergy tensor and some abelian currents) and a set of constitutive relations that reduce the number of independent degrees of freedom. Important modifications to these equations arise when external forces are applied, or when the symmetries underlying the conservation equations are anomalous. Although the standard formulation of hydrodynamic equations refers to long-wavelength deformations of finite temperature homogeneous configurations, general hydrodynamic systems can exhibit a variety of interesting extensions. These extensions can include, for instance, the presence of anisotropies, symmetries associated to higher spin currents, or the propagation of the fluid on dynamical hypersurfaces. In this paper we will encounter ideal, non-anomalous, forced hydrodynamic systems with many of these extensions.

The hydrodynamic systems that will be considered in this paper are derived from the classical long-wavelength dynamics of black holes and branes in general (super)gravity theories in the spirit of the general connection between fluids and gravity outlined above. Forced fluids have been discussed in the context of AdS black hole solutions and the fluidgravity correspondence in several papers in the past, see for instance [10]. The extension beyond AdS along the lines of the blackfold formalism introduces new ingredients and new technical complications. Let us quickly summarize some of these issues.

Forced blackfold equations in (super)gravity. In blackfold generalizations of the fluid/gravity correspondence the long-wavelength expansion of the (super)gravity equations around black brane solutions is an affair that combines the fluid dynamical nature of black hole physics [7-9, 11, 12] with the extrinsic (elastic) dynamics [7, 13-25] of hypersurfaces in ambient spacetimes that is characteristic of D-brane physics. Unfortunately, even the case of black holes in flat space is sufficiently complicated and it has not yet been studied systematically beyond the first order in the derivative expansion. The case of black holes in asymptotic spacetimes with arbitrary curved geometry and other non-trivial fluxes, which is the main case of interest in this paper, clearly involves an even more demanding technical treatment. Some of the main conceptual and technical issues that arise in this context are summarized in the discussion of section 5. In this paper we will not attempt to address a complete solution of these issues. Our main focus will remain on the leading order perturbations in supergravity aiming to isolate and determine the generic features of the effective fluid dynamical description that arise at this order.

One of the new ingredients that blackfolds introduce, and whose implications we want to emphasize here, is the simultaneous presence and interplay of different higher spin currents and background abelian gauge fields. Black holes and branes can be coupled electrically, magnetically or dyonically with respect to these gauge fields. The long-wavelength analysis of the dynamics of such solutions in arbitrary backgrounds leads to forced effective fluids with external forces that involve a variety of different couplings between the higher 
spin currents and the background gauge fields. The structure of these couplings is uniquely determined from the action of the underlying (super)gravity theory. We will perform a general analysis of this structure at the leading order of the long-wavelength expansion.

The precise identification of this structure is not only interesting as an academic exercise in fluid dynamics, it is also one of the first steps towards a systematic long-wavelength analysis of black hole solutions in general backgrounds. For example, it can be useful in problems that involve black holes in the background of curved geometries, e.g. black holes in the vicinity of other black holes, problems that involve extremal, and non-extremal, brane solutions in backgrounds with fluxes in the context of string theory (describing the gravitational backreaction of massive configurations of D/M-branes), and problems with real time dynamics where the background is forcing a black hole solution to evolve dynamically in time.

In the context of the blackfold formalism, most of the developments have focused so far on black hole solutions in flat spacetimes [16, 26, 27]. Preliminary aspects of black brane solutions in AdS spacetimes have been studied in [14, 19, 28, 29] (see also [15]). For specific AdS flux backgrounds, thermal probe brane techniques based on the blackfold approach have been applied in $[28,29]$ to construct thermal giant gravitons. However, a general treatment of blackfolds in general backgrounds with fluxes in (super)gravity theories has not been performed and part of our motivation is to initiate such study.

Another motivation for this work is the recent proposal [30] (see also [31] for related independent work building on $[17,32,33]$ ) that the effective hydrodynamic description of black brane solutions in the blackfold formalism is connected to the underlying microscopic description of $\mathrm{D} / \mathrm{M}$-branes in string/M-theory via a general open/closed string duality that works in many cases in gravity as a tomographic principle. In the proposal of ref. [30] the abelian hydrodynamic blackfold equations are conjectured to be effective equations of singleton dynamics. They provide a strong-coupling description of the effective longwavelength dynamics of the abelian, center-of-mass degrees of freedom of D/M-branes. In accordance with this expectation it was demonstrated in specific examples in [30] that the leading order blackfold equations of extremal $p$-brane configurations in string theory are equivalent to the DBI equations that describe long-wavelength dynamics of D-branes.

The formulation of the blackfold equations in backgrounds with arbitrary fluxes, that we venture here, will allow us to probe this conjecture further, in more detail and in more generic situations. As an immediate forthcoming task one can test the expected equivalence of the extremal forced blackfold equations derived in this paper with the full set of wellknown open-closed string couplings in the DBI action. The proof of this equivalence would provide a pure supergravity derivation of the complete DBI action (including all openclosed string couplings). A similar exercise could be performed in M-theory to re-derive from supergravity the PST action of M5-branes [34], which is a theory of a self-dual 3-form field. Earlier related work in this direction has appeared in [23, 35].

Brief summary of technical results and outline of the paper. The main task of this paper is to provide a general treatment of the leading order collective mode equations of $p$-brane solutions in arbitrary backgrounds for generic (super)gravity theories. We follow 
the general strategy of the blackfold approach and work in a long-wavelength derivative expansion around an exact or an approximate solution of the supergravity equations of motion (see section 5 for an explicit discussion of this distinction).

In the (super)gravity analysis of the blackfold approach one attempts to construct a perturbatively deformed black hole solution in a scheme of matched asymptotic expansions (MAEs). A separate perturbative analysis of the gravity equations is performed in the farzone (near the fixed asymptotic background), and in the near-zone (in the vicinity of the black hole horizon). At the end the solutions in the two zones are matched order-by-order in the perturbative expansion. Explicit applications of MAEs in the context of blackfolds can be found in [11-13, 22], to which we refer the reader for further details.

In section 2 we discuss how an arbitrary asymptotic background affects the constraint equations (and associated conservation equations) in the far-zone analysis. Working in the linearized approximation, in this region we describe the leading order modifications of the asymptotic geometry in the presence of a bulk source. As such, this treatment is very general and conceptually straightforward. On its own, it applies to very general black hole solutions and requires no assumptions of a long-wavelength expansion. The obtained conservation equations, which arise as Bianchi identities in gravity and abelian gauge theory, are exact and valid for generic configurations with arbitrary derivatives, in space and/or in time. We formulate them in terms of generic currents without reference to specific black holes and specific constituent relations. The key elements of the analysis are exhibited first in a simplified model of an Einstein-dilaton theory with a $(q+1)$-form gauge field. Then, the logic is applied in more general settings that include the tendimensional type-IIA/B, and eleven-dimensional supergravity theories to obtain the general forced blackfold equations in string/M-theory. Besides the standard Lorentz coupling of the schematic form $F \cdot J$ between background gauge field strengths $F$ and currents $J$, as well as their electromagnetic duals, one can also see, in the forced equations of the stress-energy tensor, couplings of the schematic form $F \cdot C \cdot J$ arising from Chern-Simons interactions in the supergravity theory. One can also see explicitly the corresponding background-dependent modifications of the conservation equations of the abelian currents.

In section 3 we initiate the study of the same equations in the near-zone region. At this point we need to be very specific about the type of solution that we consider. We can only proceed in a systematic way by setting up a perturbative expansion scheme. This is the point where the separation of scales and the small derivatives (inherent in the blackfold approach) are most needed to set up the matched asymptotic expansion that will allow us to extend the solution in the near-horizon region and to incorporate the full non-linearities of gravity beyond the linearized approximation. Generalizing earlier results for neutral black $p$-brane solutions, we consider the cases of charged black branes in Einstein-dilaton theories and D $p$-F1 bound states in type II supergravity. We focus on the constraint equations of gravity that lead to the corresponding effective blackfold equations. The $\mathrm{D} p$-F1 case is particularly interesting because it demonstrates very explicitly how couplings between currents of different spin and background fields arise simultaneously in the force terms. Of course, in all cases we reproduce the results of the asymptotic linearized treatment of section 2. The added benefit of the near-zone analysis is that it gives us a first 
taste of the ansätze that need to be implemented to find the near-zone deformations of the supergravity solutions. Eventually these solutions should be matched to the solutions of the far-zone analysis.

In section 4 we present yet another derivation of the forced blackfold equations by studying how external couplings arise in hydrostatic partition functions. After a general discussion we focus on the cases of solutions with Maxwell charge and top-form charged black $p$-brane solutions. This approach is based on the analysis of the on-shell gravitational action. It applies only to stationary configurations and leads to a natural connection with standard relations in thermodynamics. The forced blackfold equations arise in this case, in a standard fashion, as Ward identities of the on-shell gravitational action.

Finally, in section 5 we conclude with a general overview of the approach and a summarizing discussion of some of the key conceptual issues that can arise in generic longwavelength treatments of black holes in non-trivial background geometries. We point out that in ideal situations (that we dub the 'exact brane' application of blackfolds) one has exact information about a specific (leading order) homogeneous black brane configuration that is subsequently perturbed in small derivatives along the homogeneous directions. Since such exact information is mostly absent in situations with arbitrary asymptotic geometries we highlight the role that is usually played by parallel expansions of the leading order order solution. These are expansions in small numbers that are typically ratios of quantities of the leading order order solution over quantities characterizing the background. The disadvantage of this approach is the implementation of further approximations; the advantage is the possibility of a wider, more flexible application of the method. We briefly comment on the open-closed string interpretation of these additional approximations in the context of the proposal [30]. We conclude section 5 with a list of interesting open problems that could be treated in a natural continuation of this work.

We provide four appendices. In appendix A, we give the form notation that we employ throughout the paper. In appendix $\mathrm{B}$, we provide the equations of motion and probe brane equations derived in section 2 for the special case of type II A/B supergravity and in appendix $\mathrm{C}$, we provide the effective charges and currents for the brane solutions considered in sections 3 and 4. Finally, in appendix D an entropy current analysis of the forced fluids considered in section 4.3 is given.

Notation. Throughout this paper we will use Greek letters, $\mu, \nu, \ldots$, to denote the $D$ dimensional spacetime directions $x^{\mu}$, and small Latin letters, $a, b, \ldots$, to express the $p+1$ (worldvolume) directions $\sigma^{a}$, along which a $p$-brane solution is infinitely extended. The Minkowski metric is denoted by $\eta_{\mu \nu}, G$ is Newton's gravitational constant and $\star$ denotes the $D$-dimensional Hodge star operator. Further details on our notation can be found in appendix A.

\section{Far-zone analysis}

In this section we concentrate on the asymptotic region far from the black hole horizon at radial distances $r \gg r_{H}$, where $r_{H}$ is the location of the black hole horizon. In this 
region the gravitational fields are small deformations, weighted by positive powers of the small ratio $r_{H} / r$, of the asymptotic solution. In the asymptotic solution the gravitational field (and any other matter field) are assumed arbitrary. In particular, at this point, no assumption of weak field or small derivatives is made. The leading deformations induced by the black hole in the bulk can be studied in this region by analyzing the linearized Einstein equations

$$
\left.\left(G_{\mu \nu}-8 \pi G \mathbf{T}_{\mathbf{M} \mu \nu}\right)\right|_{\text {linear }}=0,
$$

where $\mathbf{T}_{\mathbf{M} \mu \nu}$ denotes the stress-energy contribution of any sources of energy/matter that are present in the gravitational theory. The subscript linear in (2.1) is there to remind that the Einstein equations are linearized around the asymptotic background.

The method of equivalent sources ${ }^{1}$ allows us to replace the complicated details of the bulk with an effective stress-energy tensor $\mathbf{T}_{\mu \nu}$ localized in the bulk

$$
\left.\left(G_{\mu \nu}-8 \pi G \mathbf{T}_{\mathbf{M} \mu \nu}\right)\right|_{\text {linear }}=8 \pi G \mathbf{T}_{\mu \nu},
$$

where $\mathbf{T}_{\mu \nu}$, which is supported on a $(p+1)$-dimensional hypersurface for a $p$-brane solution, is sourcing the gravitational field of interest at large distances. Solving (2.2) for all $r$ and extracting the result for $r \gg r_{H}$ is equivalent to solving (2.1). Using the Bianchi identity $\nabla_{\mu} G^{\mu \nu}=0$ in (2.2) we find that the total stress-energy tensor is conserved, namely

$$
\nabla_{\mu} \mathbf{T}^{\mu \nu}=-\nabla_{\mu} \mathbf{T}_{\mathbf{M}}^{\mu \nu}
$$

In what follows we will use the full set of gravitational equations to re-express the r.h.s. of this equation in terms of the asymptotic profile of the gravitational fields and other currents characterizing the source. At the end, the r.h.s. will be recast as a force term driven by the non-trivial asymptotic profiles of the gravitational fields.

The equations derived in this way from (2.3) are equations describing a probe brane in the asymptotic background. For example, when $\mathbf{T}^{\mu \nu}$ is the stress-energy tensor of a charged point particle, and $\mathbf{T}_{\mathbf{M}}^{\mu \nu}$ is the stress-energy tensor of a $\mathrm{U}(1)$ gauge field, eq. (2.3) provides a derivation of the Lorentz force acting on the particle [39]. Similar modified conservation equations of other currents will be derived from the Bianchi identities of bulk gauge fields.

In the rest of this section, we will use this strategy to derive the equations of motion of generic probe branes in Einstein-dilaton theory with a $(q+1)$-form gauge field, type IIA/B and eleven dimensional supergravity. The results cover the most general type of brane bound states that can be encountered in string theory.

The above derivation based on the linearized approximation (2.2) is (in a sense) straightforward, yet it turns out to provide very general expressions with very few assumptions. This will be most appreciated in the next section when we try to track $\mathbf{T}_{\mu \nu}$ in the bulk of the solution away from the asymptotic region. The same $\mathbf{T}_{\mu \nu}$ (and other currents) with the same modified conservation equations will arise there as constraint equations of the non-linear Einstein equations. Nevertheless, the treatment of the solution at

\footnotetext{
${ }^{1}$ See for example [36] and [37, 38].
} 
finite radius $r$ will be much harder and a systematic analysis will require more assumptions and more case-specific data.

It is also worth stressing that from the point of view of the full solution across the whole spacetime, the effective currents and their conservation equations are not merely probe data and probe equations, in fact, they are describing a full-fledged backreacted solution of the gravitational equations (see section 3).

\subsection{Einstein-dilaton theory with a $(q+1)$-form gauge field}

We start with the simplest system of Einstein-dilaton theory with a $(q+1)$-form gauge field with action

$$
I=\frac{1}{16 \pi G} \int_{\mathcal{M}_{D}}\left[\star R-\frac{1}{2} d \phi \wedge \star d \phi-\frac{1}{2} e^{a_{q} \phi} F_{q+2} \wedge \star F_{q+2}\right],
$$

where the dilaton coupling $a_{q}$ is arbitrary. We wish to couple a probe brane to field configurations which are solutions to the equations of motion that arise from (2.4). We consider a probe brane carrying an electric current $\mathbf{J}_{q+1}$, a magnetic current $\mathcal{J}_{D-q-3}$ and a dilaton current $\mathbf{j}_{\phi}$ coupled to the fields of the theory (2.4). The presence of the magnetic current modifies the Bianchi identity for the $(q+2)$-form field strength $F_{q+2}$ so that

$$
d F_{q+2}=16 \pi G \star \mathcal{J}_{D-q-3}, \quad F_{q+2}=d C_{q+1}+16 \pi G \star D_{D-q-2},
$$

where $D_{D-q-2}$ is the Dirac brane defined by $\star \mathcal{J}_{D-q-3}=d \star D_{D-q-2}$. The equations of motion for the gauge field $C_{q+1}$ and the dilaton $\phi$ for the theory (2.4) coupled to sources are

$$
\begin{aligned}
d \star\left(e^{a_{q} \phi} F_{q+2}\right) & =(-1)^{D+q^{2}-1} 16 \pi G \star \mathbf{J}_{q+1}, \\
\square \phi-\frac{a_{q}}{2} e^{a_{q} \phi} \star\left(F_{q+2} \wedge \star F_{q+2}\right) & =-16 \pi G \mathbf{j}_{\phi} .
\end{aligned}
$$

In order to evaluate the r.h.s. of eq. (2.3) we require the explicit form of the energy/matter contributions to $\mathbf{T}_{\mathbf{M}}^{\mu \nu}$. Splitting $\mathbf{T}_{\mathbf{M}}^{\mu \nu}$ as $\mathbf{T}_{\mathbf{M}}^{\mu \nu}=\mathbf{T}_{(F)}^{\mu \nu}+\mathbf{T}_{(\phi)}^{\mu \nu}$ we obtain the bulk stress-energy tensor for the $(q+1)$-form gauge field and the dilaton

$$
\begin{aligned}
16 \pi G \mathbf{T}_{(F)}^{\mu \nu} & =\frac{e^{a_{q} \phi}}{(q+1) !}\left(F_{q+2}^{\mu \mu_{1} \ldots \mu_{q+1}} F_{q+2}{ }^{\nu}{ }_{\mu_{1} \ldots \mu_{q+1}}-\frac{1}{2(q+2)} g^{\mu \nu} F_{q+2}^{2}\right), \\
16 \pi G \mathbf{T}_{(\phi)}^{\mu \nu} & =\partial^{\mu} \phi \partial^{\nu} \phi-\frac{1}{2} g^{\mu \nu} \partial_{\lambda} \phi \partial^{\lambda} \phi .
\end{aligned}
$$

Inserting these expressions into the r.h.s. of eq. (2.3) we find

$$
\begin{aligned}
16 \pi G \nabla_{\mu} \mathbf{T}_{\mathbf{M}}^{\mu \nu}= & \frac{1}{(q+1) !} F_{q+2}^{\nu \mu_{1} \ldots \mu_{q+1}} \nabla_{\mu}\left(e^{a_{q} \phi} F_{q+2 \mu_{1} \ldots \mu_{q+1}}^{\mu}\right)-\frac{e^{a_{q} \phi}}{(q+2) !} F_{q+2}^{\mu_{1} \ldots \mu_{q+2}} d F^{\nu}{ }_{q+2 \mu_{1} \ldots \mu_{q+2}} \\
& +\left(\square \phi-\frac{a_{q}}{2} e^{a_{q} \phi} \star\left(F_{q+2} \wedge \star F_{q+2}\right)\right) \partial^{\nu} \phi .
\end{aligned}
$$

This result simplifies further by using the equations of motion (2.6). Inserting the final expression into (2.3) leads to the modified conservation equation

$$
\begin{aligned}
\nabla_{\mu} \mathbf{T}^{\mu \nu}= & \frac{1}{(q+1) !} F_{q+2}^{\nu \mu_{1} \ldots \mu_{q+1}} \mathbf{J}_{q+1 \mu_{1} \ldots \mu_{q+1}} \\
& +\frac{(-1)^{q D+1} e^{a_{q} \phi}}{(D-q-3) !} F_{D-q-2}^{\nu \mu_{1} \ldots \mu_{D-q-3}} \mathcal{J}_{D-q-3 \mu_{1} \ldots \mu_{D-q-3}}+\mathbf{j}_{\phi} \partial^{\nu} \phi
\end{aligned}
$$


where we have defined the dual field strength via the relation $F_{D-q-2}=\star F_{q+2}$. In the case of a non-dilatonic electrically charged point-particle $(q=0)$, eq. (2.9) yields the Lorentz force. In general, eq. (2.9) describes the electric coupling to the field strength $F_{q+2}$ and to its dual $F_{D-q-2}$. In addition, we obtain from general principles the force due to the presence of background dilaton fields, which is proportional to the gradient of $\phi$. This type of force has been encountered previously, for example, in the context of forced fluid dynamics [10] in the fluid/gravity correspondence.

The conservation equations for the currents $\mathbf{J}_{q+1}$ and $\mathcal{J}_{D-q-3}$ can be obtained similarly as Bianchi identities from the first equation in (2.5) and (2.6). They yield

$$
\begin{aligned}
d \star \mathbf{J}_{q+1} & =0, \\
d \star \mathcal{J}_{D-q-3} & =0 .
\end{aligned}
$$

The dilaton current $\mathbf{j}_{\phi}$ does not obey any conservation equation, as can be seen from its equation of motion (2.6).

The dilaton in the case above (and all others considered in this paper) describes primary hair. We remark that in some setups there can be extra equations, that do not originate from conservation equations, but as a consequence of boundary conditions. This is for example the case in forced superfluid dynamics considered in [40] where the conservation equations are supplemented by an extra equation coming from requiring AdS asymptotics, which in the dual fluid description becomes the zero curl condition on the superfluid velocity.

Equations for localized stress-energy tensor and currents. The equations of motion (2.9)-(2.10) were obtained for arbitrary stress-energy tensor and currents. However, in most of this work we are interested in localized stress-energy tensor and currents describing a $(p+1)$-dimensional probe. In this particular case,

$$
\begin{aligned}
\mathbf{T}^{\mu \nu} & =T^{\mu \nu} \tilde{\delta}^{(n+2)}\left(x^{\mu}-X^{\mu}\right), & \mathbf{J}_{q+1} & =J_{q+1} \tilde{\delta}^{(n+2)}\left(x^{\mu}-X^{\mu}\right), \\
\mathcal{J}_{D-q-3} & =\mathcal{J}_{D-q-3} \tilde{\delta}^{(n+2)}\left(x^{\mu}-X^{\mu}\right), & \mathbf{j}_{\phi} & =j_{\phi} \tilde{\delta}^{(n+2)}\left(x^{\mu}-X^{\mu}\right),
\end{aligned}
$$

where $\tilde{\delta}^{(n+2)}\left(x^{\mu}-X^{\mu}\right)$ is the reparametrization invariant delta function localized in the $(n+2)=(D-p-1)$-transverse directions, $x^{\mu}$ are spacetime coordinates and $X^{\mu}$ the set of mapping functions describing the position of the object in the ambient spacetime with metric $g_{\mu \nu}$. All indices in (2.11) are tangential to the probe's worldvolume $\mathcal{W}_{p+1}$, e.g., $T^{\mu \nu}=T^{a b} \partial_{a} X^{\mu} \partial_{b} X^{\nu}$ where $\partial_{a} X^{\mu}$ acts as a projector onto the worldvolume. Projecting eq. (2.9) along the worldvolume one obtains

$$
\begin{aligned}
\nabla_{a} T^{a b}= & \frac{1}{(q+1) !} \mathcal{F}_{q+2}^{b a_{1} \ldots a_{q+1}} J_{q+1 a_{1} \ldots a_{q+1}} \\
& +\frac{(-1)^{q D+1} e^{a_{q} \phi}}{(D-q-3) !} \mathcal{F}_{D-q-2}^{b a_{1} \ldots a_{D-q-3}} \mathcal{J}_{D-q-3 a_{1} \ldots a_{D-q-3}}+j_{\phi} \partial^{b} \varphi
\end{aligned}
$$

expressing the conservation of the stress-energy tensor along the worldvolume. Here, and in the following, we have introduced $\mathcal{F}_{q+2}, \varphi$ to denote the pull-back of the background fields 
$F_{q+2}, \phi$ onto the worldvolume of the brane. Defining the transverse projector $n^{i}{ }_{\mu}$ onto the transverse $(n+2)$-dimensional space such that $n_{\mu}^{i} \partial_{a} X^{\mu}=0$ we can project eq. (2.9) along the transverse direction to obtain

$$
\begin{aligned}
T^{a b} K_{a b}{ }^{i}= & \frac{1}{(q+1) !} \mathcal{F}_{q+2}^{i a_{1} \ldots a_{q+1}} J_{q+1 a_{1} \ldots a_{q+1}} \\
& +\frac{(-1)^{q D+1} e^{a_{q} \phi}}{(D-q-3) !} \mathcal{F}_{D-q-2}^{i a_{1} \ldots a_{D-q-3}} \mathcal{J}_{D-q-3 a_{1} \ldots a_{D-q-3}}+j_{\phi} \partial^{i} \varphi,
\end{aligned}
$$

where $K_{a b}{ }^{i}=n_{\mu}^{i} \nabla_{a} \partial_{b} X^{\mu}$ is the extrinsic curvature of the worldvolume. Eq. (2.13) expresses the mechanical balance of forces on the worldvolume.

Finally, the current conservation equations (2.10) lead to

$$
\nabla_{a_{1}} J_{q+1}^{a_{1} \ldots a_{q+1}}=0, \quad \nabla_{a_{1}} \mathcal{J}_{D-q-3}^{a_{1} \ldots a_{D-q-3}}=0,
$$

which express the conservation of electric and magnetic currents along the worldvolume. ${ }^{2}$

So far we do not assume any specific form for the currents. In section 3 we will present explicit cases of these equations that arise as constraint equations for perturbations of particular types of black brane solutions.

\subsection{Type IIA/B supergravity}

Next we consider the slightly more non-trivial cases of type IIA/B supergravity. Both of these cases can be treated in the same framework using the democratic formulation introduced in [41], in which the number of degrees of freedom of the RR sector are doubled. ${ }^{3}$ The action for the bosonic sector of ten-dimensional supergravity in the Einstein frame can be written as [41],

$$
I=\frac{1}{16 \pi G} \int_{\mathcal{M}_{10}}\left[\star R-\frac{1}{2} d \phi \wedge \star d \phi-\frac{1}{2} e^{-\phi} H_{3} \wedge \star H_{3}-\frac{1}{4} \sum_{q} e^{a_{q} \phi} \tilde{F}_{q+2} \wedge \star \tilde{F}_{q+2}\right],
$$

where the dilaton coupling $a_{q}$ is given by $a_{q}=(3-q) / 2$ and $D=10$. Here the index $q$ runs over the values $q=0,2,4,6$ for type IIA ${ }^{4}$ and the values $q=-1,3,5,7$ for type IIB.

In this theory, we consider a brane probe with electric current $\mathbf{j}_{2}$, which sources the NSNS field strength $H_{3}$, and a set of electric currents $\mathbf{J}_{q+1}$, which source the RR field strengths $\tilde{F}_{q+2}$. We also assume that the brane is characterized by a dilatonic current $\mathbf{j}_{\phi}$ and several magnetic currents, namely, a non-zero magnetic current $\mathfrak{j}_{6}$, which modifies the Bianchi identity for $H_{3}$, and a set of magnetic currents $\mathcal{J}_{D-q-3}$, which modify the Bianchi identities for the RR fields, i.e.,

$$
\begin{aligned}
d H_{3} & =16 \pi G \star \mathfrak{j}_{6}, \\
d \tilde{F}_{q+2}-H_{3} \wedge \tilde{F}_{q} & =16 \pi G\left(\star \mathcal{J}_{D-q-3}-\left(\star \mathbf{j}_{6} \wedge C_{q-1}\right)\right) .
\end{aligned}
$$

\footnotetext{
${ }^{2}$ We have not assumed the existence of any boundaries $\partial \mathcal{W}_{p+1}$. In case they are present these equations must be supplemented by the boundary conditions $T^{a b} \eta_{a}=J_{q+1}^{a_{1} \ldots a_{q+1}} \eta_{a_{1}}=\left.\mathcal{J}_{D-q-3}^{a_{1} \ldots a_{D-q-3}} \eta_{a_{1}}\right|_{\partial \mathcal{W}_{p+1}}=0$.

${ }^{3}$ One may consider the more general case in which the degrees of freedom of the NSNS sector are also doubled [42, 43], however, these formulations introduce several new structures which are not required for the purposes of this paper.

${ }^{4}$ We are not including here the case $q=8$, however, the results presented in this section could be easily generalized to include this case.
} 
This implies that the field strengths $H_{3}$ and $\tilde{F}_{q+2}$ are given by the expressions

$$
H_{3}=d B_{2}+16 \pi G \star \mathcal{D}_{7}, \quad \tilde{F}_{q+2}=F_{q+2}-H_{3} \wedge C_{q-1},
$$

where we have defined $F_{q+2}=d C_{q+1}+16 \pi G \star D_{D-q-2}$. We have introduced the Dirac brane $\mathcal{D}_{7}$ satisfying $\star \mathbf{j}_{6}=d \star \mathcal{D}_{7}$, as well as the Dirac branes $D_{D-q-2}$ satisfying $\star \mathcal{J}_{D-q-3}=$ $d \star D_{D-q-2}$. One may now couple the currents of the charged probe brane to the fields of the theory (2.15) via the sourced equations of motion

$$
\begin{aligned}
& d\left(\star\left(e^{-\phi} H_{3}\right)-\sum_{q} \frac{1}{2 !} e^{a_{q} \phi}\left[\star \tilde{F}_{q+2} \wedge C_{q-1}\right]\right)=-16 \pi G \star \mathbf{j}_{2}, \\
& d \star\left(e^{a_{q}} \tilde{F}_{q+2}\right)+(-1)^{q} e^{a_{q+2} \phi}\left[\star \tilde{F}_{q+4} \wedge H_{3}\right]=(-1)^{q} 16 \pi G \star \mathbf{J}_{q+1}, \\
& \square \phi+\frac{1}{2} e^{-\phi} \star\left(H_{3} \wedge \star H_{3}\right)-\sum_{q} \frac{a_{q}}{4} e^{a_{q} \phi} \star\left(\tilde{F}_{q+2} \wedge \star \tilde{F}_{q+2}\right)=-16 \pi G \mathbf{j}_{\phi} .
\end{aligned}
$$

We note that the second equation in (2.18) is in fact a set of equations, one for each $q$ present in (2.15). As noted in [41], the action (2.15) is a pseudo-action and the equations of motion (2.18) must be supplemented by the duality relations

$$
\tilde{F}_{q+2}=(-1)^{[q / 2]+1} e^{-a_{q} \phi} \star \tilde{F}_{D-q-2}
$$

in order to account for the correct number of degrees of freedom. Once imposing (2.19), the equations of motion for the higher-form fields $\tilde{F}_{q+2}$ with $q=4, \ldots, 7$ yield the Bianchi identities (2.16) for the lower form fields $q=-1, \ldots, 3$, while the Bianchi identities for the higher-form fields yield the equations of motion for the lower form fields. This means that the 8 currents ( 4 electric and 4 magnetic) associated with the higher-form fields are given in terms of the currents associated with the lower form fields, i.e.,

$$
\begin{aligned}
\star \mathbf{J}_{D-q-3} & =(-1)^{q+1+[q / 2]}\left(\star \mathcal{J}_{D-q-3}-\left(\star \mathbf{j}_{6} \wedge C_{q-1}\right)\right), \\
\star \mathbf{J}_{q+1} & =(-1)^{[q / 2]}\left(\star \mathcal{J}_{q+1}-\left(\star \mathbf{j}_{6} \wedge C_{D-q-5}\right)\right), \quad q=4 \ldots, 7,
\end{aligned}
$$

and furthermore, due to the self-duality relation $\tilde{F}_{5}=\star \tilde{F}_{5}$ we also have that

$$
\star \mathbf{J}_{4}=-\left(\star \mathcal{J}_{4}-\left(\star \mathbf{j}_{6} \wedge C_{2}\right)\right)
$$

Using the explicit form of the stress-energy tensor contributions (2.7), with appropriate factors ${ }^{5}$ one may readily derive as before the equations of motion of a probe brane in type

\footnotetext{
${ }^{5}$ The stress-energy tensor for the RR fields in the action (2.15) is given by $1 / 2$ of (2.7) due to the $1 / 4$ factor in the action (2.15) for the RR fields.
} 
IIA/B supergravity. The final expression is

$$
\begin{aligned}
\nabla_{\mu} \mathbf{T}^{\mu \nu}= & \frac{1}{2 !} H_{3}^{\nu \mu_{1} \mu_{2}} \mathbf{j}_{2 \mu_{1} \mu_{2}}+\frac{e^{-\phi}}{6 !} H_{7}^{\nu \mu_{1} \ldots \mu_{6}} \mathbf{j}_{6 \mu_{1} \ldots \mu_{6}}+\mathbf{j}_{\phi} \partial^{\nu} \phi \\
& +\sum_{q} \frac{1}{(q+1) !}\left(\tilde{F}_{q+2}^{\nu \mu_{1} \ldots \mu_{q+1}}+(-1)^{q+1} \frac{q(q+1)}{2 !} H_{3}^{\nu \mu_{1} \mu_{2}} C_{q-1}^{\mu_{3} \ldots \mu_{q+1}}\right) \mathbf{J}_{q+1 \mu_{1} \ldots \mu_{q+1}} \\
& +\sum_{q} \frac{e^{a_{q} \phi}}{(\tilde{q}+1) !}\left(\tilde{F}_{\tilde{q}+2}^{\nu \mu_{1} \ldots \mu_{\tilde{q}+1}}+(-1)^{\tilde{q}+1} \frac{\tilde{q}(\tilde{q}-1)}{2 !} H_{3}^{\nu \mu_{1} \mu_{2}} C_{\tilde{q}-1}^{\mu_{3} \ldots \mu_{q+1}}\right) \mathcal{J}_{\tilde{q}+1} \mu_{\mu_{1} \ldots \mu_{\tilde{q}+1}} \\
& +\frac{1}{4 !}\left(\tilde{F}_{5}^{\nu \mu_{1} \ldots \mu_{4}}+3 H_{3}^{\nu \mu_{1} \mu_{2}} C_{2}^{\mu_{3} \ldots \mu_{4}}\right) \mathbf{J}_{4 \mu_{1} \ldots \mu_{4}} \\
& \left.-\sum_{q} \frac{e^{a_{q} \phi}}{(q+2) !} \tilde{F}_{q+2}^{\mu_{1} \ldots \mu_{q+2}\left[\star \mathbf{j}_{6}\right.} \wedge C_{q-1}\right]_{\mu_{1} \ldots \mu_{q+2}}^{\nu}
\end{aligned}
$$

where we have introduced the dual of $H_{3}$ via the relation $H_{7}=\star H_{3}$ and the duals $\tilde{F}_{D-q-2}=$ $\star \tilde{F}_{q+2}$. The sums over $q$ take values only over $q=-1, \ldots, 2$ and we have introduced $\tilde{q}=D-q-4$. Note that since we have imposed the duality conditions (2.19) we assume in (2.19) that $C_{q}=0$ for $q \geq 3$. The type of force terms involved here include Lorentz forces due to the presence of $H_{3}, H_{7}, F_{q+2}$ and $\tilde{F}_{\tilde{q}+2}$, the force term due to the non-trivial dilaton as well as several force term originating from Chern-Simons terms.

One can also obtain the conservation equations for the remaining currents using eqs. (2.16) and (2.18). They are

$$
\begin{aligned}
& d \star \mathbf{J}_{q+1}+(-1)^{q+1} \star \mathbf{J}_{q+3} \wedge H_{3}+(-1)^{q+1} e^{a_{q+2} \phi} \star \tilde{F}_{q+4} \wedge \star \mathbf{j}_{6}=0, \quad q=0, \ldots, 3 \\
& d \star \mathcal{J}_{D-q-3}=0, \quad q=-1,0, \quad d \star \mathcal{J}_{D-q-3}=H_{3} \wedge \star \mathcal{J}_{D-q-1}, \quad q=1,2, \\
& d \star \mathbf{j}_{2}=0, \quad d \star \mathbf{j}_{6}=0 .
\end{aligned}
$$

The dilaton current $\mathbf{j}_{\phi}$ does not obey any conservation equation neither does the current $\mathbf{J}_{(0)}$.

The reader can find the more specific form of the above expressions for each of the type IIA/B cases explicitly in appendix B.

\subsection{Eleven-dimensional supergravity}

Finally we consider probe branes coupled to field configurations which are solutions of the eleven-dimensional supergravity action $[44]^{6}$

$$
I=\frac{1}{16 \pi G} \int_{\mathcal{M}_{11}}\left[\star R-\frac{1}{2} F_{4} \wedge \star F_{4}-\frac{1}{6} \int C_{3} \wedge F_{4} \wedge F_{4}\right] .
$$

The probe branes are assumed to carry an electric current $\mathbf{J}_{3}$ and a magnetic current $\mathcal{J}_{6}$. The presence of the magnetic current modifies the Bianchi identity for the field strength $F_{4}$ so that

$$
d F_{4}=16 \pi G \star \mathcal{J}_{6}, \quad F_{4}=d C_{3}+16 \pi G \star D_{7},
$$

\footnotetext{
${ }^{6}$ It is possible, instead, to use the democratic formulation of eleven-dimensional supergravity [45] but this necessitates introducing auxiliary variables. While such endeavor might be of interest, it goes beyond the purposes of this paper.
} 
where we have introduced the Dirac brane $D_{7}$ satisfying $\star \mathcal{J}_{6}=d \star D_{7}$. The electric coupling modifies the supergravity equations of motion

$$
d \star F_{4}+\frac{1}{2}\left(F_{4} \wedge F_{4}\right)=-16 \pi G \star \mathbf{J}_{3} .
$$

Repeating the procedure of the previous sections, we deduce the following modified conservation equations

$$
\begin{aligned}
\nabla_{\mu} \mathbf{T}^{\mu \nu} & =\frac{1}{3 !} F_{3}^{\nu \mu_{1} \mu_{2} \mu_{3}} \mathbf{J}_{3 \mu_{1} \mu_{2} \mu_{3}}+\frac{1}{6 !} F_{7}^{\nu \mu_{1} \ldots \mu_{6}} \mathcal{J}_{6 \mu_{1} \ldots \mu_{6}}, \\
d \star \mathbf{J}_{3}+\star \mathcal{J}_{6} \wedge F_{4} & =0, \\
d \star \mathcal{J}_{6} & =0,
\end{aligned}
$$

where we have defined the dual field strength $F_{7}$ such that $F_{7}=\star F_{4}$. In contrast with the type IIA/B cases, here the force equations (2.27) include only the Lorentz force terms.

\section{$3 \quad$ Near-zone analysis}

We are now in position to move to the more involved part of our analysis. Our main task is to study the gravitational equations in the bulk near the horizon of the putative solution, and to identify there the role of the modified conservation equations of the previous section. This entails concrete information about the form of the bulk solution and the physics near the horizon. In order to proceed systematically it is convenient to restrict the scope of the exercise in two ways.

First, it is useful to consider solutions that are long-wavelength deformations of a leading order homogeneous solution (with the proper asymptotics at infinity). If $\mathcal{R}$ is the characteristic length scale of the deformation, then, by assumption, we consider the longwavelength regime $r_{H} \ll \mathcal{R}$ where $r_{H}$ is the smallest scale associated to the brane (such as the horizon size). ${ }^{7}$ The near-zone analysis focuses on the region $r \ll \mathcal{R}$. In the discussion of section 5 we refer to this long-wavelength expansion as the 'exact brane' application of blackfolds. This expansion assumes an exact (partially) homogeneous solution of the gravitational equations with the required large- $r$ asymptotics.

Since an exact leading order solution is not always known it is frequently useful to resort to a further parallel expansion of the leading order solution in powers of the small ratio $r_{H} / L$, where $L$ is the characteristic length scale of the background. In this regime, $r_{H} \ll \mathcal{R}, L$, the perturbative expansion assumes small derivatives not only of the black brane solution we are searching for, but also small derivatives of the asymptotic background solution. At leading order in $r_{H} / L$ we can approximate the leading order solution in the near-zone region by a black $p$-brane solution in flat space. That is the basis of a concrete ansatz for the gravitational fields in the near-zone region. Further qualitative features of the above two expansions are reviewed and re-discussed in the section 5 .

\footnotetext{
${ }^{7}$ More precisely, in order to determine the regime of validity of these long-wavelength deformations, it is required that the magnitude of all scalar invariants (including scalars associated with the background) appearing in an effective action at a given perturbative order is much smaller than those of the preceding orders. Specific details for stationary perturbations and uncharged branes can be found in [26].
} 
In this section we proceed with the assumption of the double-perturbative regime $r_{H} / \mathcal{R} \ll 1, r_{H} / L \ll 1$, and determine the role of the modified conservation equations at leading order in the perturbation. We show in specific examples that equations like (2.9) arise as constraint equations in gravity in exact analogy to the derivations in the fluid/gravity correspondence. Conservation equations of the type (2.12) and (2.14) arise from worldvolume (intrinsic) perturbations of the brane solution, while the equilibrium equations (2.13), that determine $X^{\mu}$, arise from elastic-type (extrinsic) perturbations which break the symmetries of the transverse space to the worldvolume. We consider separately both types of perturbations.

Extending the analysis of [22] for neutral branes we treat in this section the case of perturbations of dilatonic black $p$-branes charged under a $(p+1)$-form gauge potential and the $\mathrm{D} p$-F1 bound state in type II string theory, both in the presence of external background fields. These two representative cases serve as illustrative examples, which can be further extended to more complicated brane configurations and/or to theories with additional matter fields.

\subsection{Constraint equations for charged black branes}

We start by considering the example of charged $p$-brane solutions in theories of gravity with spacetime action (2.4) with $q=p$. Besides the metric, the theory includes a dilaton and a $(p+1)$-form gauge field. We focus on the class of charged dilatonic $p$-brane solutions to the equations of motion that arise from (2.4) obtained in [46]. Incorporating a boost velocity $u^{a}$ on the worldvolume, the metric, dilaton $\phi$ and the $(p+1)$-form gauge field $C_{p+1}$ of the corresponding charged $p$-brane take the form

$$
\begin{aligned}
\mathrm{d} s^{2} & =H^{-\frac{N n}{D-2}}\left(P_{a b}-f u_{a} u_{b}\right) \mathrm{d} \sigma^{a} \mathrm{~d} \sigma^{b}+H^{\frac{N(p+1)}{D-2}}\left(f^{-1} \mathrm{~d} r^{2}+r^{2} \mathrm{~d} \Omega_{(n+1)}^{2}\right), \\
\phi & =\frac{a_{p} N}{2} \log H, \quad C_{p+1}=\sqrt{N} \operatorname{coth} \alpha\left(H^{-1}-1\right) \star_{(p+1)} 1 .
\end{aligned}
$$

Here $P_{a b}=\eta_{a b}+u_{a} u_{b}$ is the projector on the worldvolume in directions orthogonal to the constant unit timelike vector $u^{a}$, while ${ }_{(p+1)} 1=\mathrm{d} t \wedge \mathrm{d} x^{1} \wedge \ldots \wedge \mathrm{d} x^{p}$ is the induced volume form on the worldvolume. The functions $f \equiv f(r)$ and $H \equiv H(r)$ are given by

$$
f(r)=1-\left(\frac{r_{0}}{r}\right)^{n}, \quad H(r)=1+\left(\frac{r_{0}}{r}\right)^{n} \sinh ^{2} \alpha .
$$

The dilaton coupling constant is arbitrary and related to $N, p, D$ through

$$
n=D-p-3, \quad a_{p}^{2}=\frac{4}{N}-\frac{2(p+1) n}{D-2} .
$$

The effective currents and charges for this particular solution are given in appendix C.1.

We have also introduced the induced metric on the worldvolume $\eta_{a b}=\eta_{\mu \nu} \partial_{a} X^{\mu} \partial_{b} X^{\nu}$ with trivial embedding scalars $X^{\mu}$, such that $\partial_{a} X^{\mu}=\delta_{a}^{\mu}$. If the embedding scalars $X^{\mu}$, which determine the position of the worldvolume geometry, are non-trivial, they will induce a spontaneous breaking of the isometry group of the asymptotic background solution. In the case of flat space we have the breaking

$$
\mathrm{SO}(1, D-1) \rightarrow \mathrm{SO}(1, p) \times \mathrm{SO}(n+2) .
$$


Leading order ansatz in the presence of background fields. Starting from the exact solution above, we wish to construct classes of solutions to the action (2.4) that asymptote at large $r$ to a given background solution

$$
\mathrm{d} s^{2}=g_{\mu \nu}(x) \mathrm{d} x^{\mu} \mathrm{d} x^{\nu}, \quad \phi(x), \quad C_{p+1}(x) .
$$

Here, the asymptotic background fields $g_{\mu \nu}, \phi$ and $C_{p+1}$ are a priori arbitrary profiles. We now assume that we put a brane in this background with embedding coordinates $X^{\mu}(\sigma)$ and that the (shortest) length scale $L$ characterizing the background is much larger than the (largest) length scale $r_{H}$ that enters the black brane solution (3.1), i.e. $r_{H} \ll L$. Thus to leading order we can ignore the variations of the background fields close to the brane. Given the brane with embedding coordinates $X^{\mu}(\sigma)$ and a foliation in the transverse coordinates, which we denote collectively by $y$, we can parametrize the spacetime with coordinates $X^{\mu}(\sigma, y)$ where we choose $X^{\mu}(\sigma, 0)=X^{\mu}(\sigma)$. This means that in the overlap region, i.e. the asymptotic region of the brane, the background can be approximated as

$$
\mathrm{d} s^{2} \simeq \gamma_{a b}(\sigma, y) \mathrm{d} \sigma^{a} \mathrm{~d} \sigma^{b}+\mathrm{d} r^{2}+r^{2} \mathrm{~d} \Omega_{(n+1)}^{2}, \quad \phi \simeq \varphi(\sigma, y), \quad C_{p+1} \simeq \mathcal{C}_{p+1}(\sigma, y) .
$$

Here

$$
\gamma_{a b}(\sigma, y)=\partial_{a} X^{\mu} \partial_{b} X^{\nu} g_{\mu \nu}(X(\sigma, y)), \quad \varphi(\sigma, y)=\phi(X(\sigma, y)), \quad \mathcal{C}_{p+1}(\sigma, y)=C_{p+1}(X(\sigma, y))
$$

are the pull-backs of the background fields (3.4) onto the foliated hypersurfaces spanning the spacetime. In particular for suitable chosen transverse coordinates (see below) at $y=0$ these expressions are the pull-back onto the worldvolume $\mathcal{W}_{p+1}$ of the brane.

To leading order we can make an ansatz for the full solution that at short distances is described by (3.1) and at large distances by (3.4). This can be achieved by considering perturbations to the exact solution (3.1) in which we promote the free constant parameters $r_{0}, \alpha, u^{a}, \partial_{a} X^{\mu}$ to slowly varying functions of the worldvolume coordinates $\sigma^{a}$, providing the leading order ansatz

$$
\begin{aligned}
\mathrm{d} s^{2} & =H^{-\frac{N n}{D-2}}\left(P_{a b}(\sigma, y)-f u_{a}(\sigma) u_{b}(\sigma)\right) \mathrm{d} \sigma^{a} \mathrm{~d} \sigma^{b}+H^{\frac{N(p+1)}{D-2}}\left(f^{-1} \mathrm{~d} r^{2}+r^{2} \mathrm{~d} \Omega_{(n+1)}^{2}\right), \\
C_{p+1} & =\mathcal{C}_{p+1}(\sigma, y)+e^{-a_{p} \varphi(\sigma, y) / 2} \sqrt{N} \operatorname{coth} \alpha(\sigma)\left(H^{-1}-1\right) \star_{(p+1)} 1, \\
\phi & =\varphi(\sigma, y)+\frac{a_{p} N}{2} \log H .
\end{aligned}
$$

Here $P_{a b}(\sigma, y)=\gamma_{a b}(\sigma, y)+u_{a}(\sigma) u_{b}(\sigma)$ and $\gamma_{a b}(\sigma, y), \mathcal{C}_{p+1}(\sigma, y), \varphi(\sigma, y)$ are the pull-backs of the background fields given in (3.6). We also note that the functions $f$ and $H$ depend on $\sigma$ through their dependence on $r_{0}, \alpha$. Furthermore, we have only kept the $\sigma$-dependence in $u^{a}(\sigma)$, absorbing possibly $y$-dependent terms into higher order corrections to the metric.

This ansatz has the property that it asymptotes at large $r$ to the background (3.5) in the overlap region and, moreover it solves the equations of motion with constant $\gamma_{a b}=$ $\eta_{a b}, \mathcal{C}_{p+1}, u^{a}, \varphi, r_{0}$, and $\alpha$ as it essentially reduces to (3.1). To see this, note first that we can always add a constant $\mathcal{C}_{p+1}$ to the gauge field $C_{p+1}$ as it corresponds to a gauge transformation and second that we can always shift the dilaton with a constant $\varphi$ 
if we also rescale the field strength with a constant factor $e^{-a_{p} \varphi / 2}$. Indeed, both of these transformations correspond to symmetries of the action (2.4).

Once the brane parameters are promoted to functions of $\sigma^{a}$ as is done in the ansatz (3.7), it is necessary to add corrections to all of the fields, which are determined by solving the equations of motion of the action. In a derivative expansion, these corrections can be of two types: intrinsic and extrinsic. To first order, intrinsic corrections are derivative corrections in the brane parameters $r_{0}, \alpha, u^{a}, \varphi$. Denoting $\lambda$ as the minimum length scale associated with fluctuations of these fields, we introduce the small expansion parameter $\varepsilon=r_{H} / \lambda \ll 1$ which controls intrinsic perturbations.

Extrinsic perturbations, in turn, are fluctuations in the extrinsic geometry of the brane worldvolume. To first order they appear due to the non-zero extrinsic curvature $K_{a b}{ }^{i}$ of the induced metric. Denoting $\mathcal{R}$ as the length scale associated to such fluctuations we introduce the expansion parameter $\tilde{\varepsilon}=r_{H} / \mathcal{R} \ll 1$ which controls extrinsic perturbations. In general, the corrections appear as a multipole expansion in the transverse sphere $S^{(n+1)}$. For example, for the metric perturbation, we can write to first order in derivatives

$$
h_{\mu \nu}(r, \theta)=\varepsilon f_{\mu \nu}(r)+\tilde{\varepsilon} \cos \theta d_{\mu \nu}(r)+\ldots,
$$

where $f_{\mu \nu}(r)$ is the monopole part and purely intrinsic and $d_{\mu \nu}(r)$ is the dipole part and purely extrinsic. Thus at first order intrinsic and extrinsic deformations decouple, which will be used below. To higher orders the two types of perturbations couple generically. It is important to stress that our focus will not be to determine the corrections $h_{\mu \nu}$ (and the corresponding ones for the gauge field and dilaton), but instead to extract the subset of field equations that are exactly independent of these corrections.

Intrinsic equations. We first consider the intrinsic perturbations $f_{\mu \nu}$, which do not break the symmetries of the transverse space, i.e. $\partial_{a} X^{\mu}=\delta_{a}^{\mu}$ so that the induced metric becomes trivial. It follows that the leading order background metric (3.5) in the overlap region is given by

$$
\mathrm{d} s^{2}=\eta_{a b} \mathrm{~d} \sigma^{a} \mathrm{~d} \sigma^{b}+\mathrm{d} r^{2}+r^{2} \mathrm{~d} \Omega_{(n+1)}^{2}, \quad \phi=\varphi(\sigma), \quad C_{p+1}=\mathcal{C}_{p+1}(\sigma),
$$

and we proceed by considering the derivative expansion of the leading order solution (3.7). Because the background fields only depend on the worldvolume coordinates $\sigma^{a}$, the intrinsic perturbations do not couple to extrinsic perturbations. The analysis of these perturbations is very similar to that of the well-known fluid/gravity correspondence [10] (see also [47, 48] which treats the case for charged branes).

The expansion is controlled by the parameter $\varepsilon$ which is assumed to be of the order of the inverse length scale of the variation of the fields $u^{a}, \varphi, r_{0}$, and $\alpha$ over the length scale $\lambda$ of the fluctuations. We can then write, up to first order, the expansions

$$
\mathrm{d} s^{2}=\mathrm{d} s_{(0)}^{2}+\varepsilon f_{\mu \nu} \mathrm{d} x^{\mu} \mathrm{d} x^{\nu}, \quad \phi=\phi^{(0)}+\varepsilon \phi^{(1)}, \quad C_{p+1}=C_{p+1}^{(0)}+\varepsilon C_{p+1}^{(1)},
$$

where we regard (3.7) as the first term in the expansion, i.e., as $\mathrm{d} s_{(0)}^{2}, \phi^{(0)}$, and $C_{p+1}^{(0)}$ and the (intrinsic) fields $u^{a}, \varphi, r_{0}$, and $\alpha$ are expanded at a given point $\mathcal{P}$ such that

$$
\begin{array}{rlrl}
u^{a}(\sigma) & =\left.u^{a}\right|_{\mathcal{P}}+\left.\varepsilon \sigma^{b} \partial_{b} u^{a}\right|_{\mathcal{P}}+\mathcal{O}\left(\varepsilon^{2}\right), & & \varphi(\sigma)=\left.\varphi\right|_{\mathcal{P}}+\left.\varepsilon \sigma^{a} \partial_{a} \varphi\right|_{\mathcal{P}}+\mathcal{O}\left(\varepsilon^{2}\right) \\
r_{0}(\sigma)=\left.r_{0}\right|_{\mathcal{P}}+\left.\varepsilon \sigma^{a} \partial_{a} r_{0}\right|_{\mathcal{P}}+\mathcal{O}\left(\varepsilon^{2}\right), & & \alpha(\sigma)=\left.\alpha\right|_{\mathcal{P}}+\left.\varepsilon \sigma^{a} \partial_{a} \alpha\right|_{\mathcal{P}}+\mathcal{O}\left(\varepsilon^{2}\right) .
\end{array}
$$


We note that since $\mathcal{C}_{p+1}$ does not depend on the transverse space coordinates it simply corresponds to a gauge transformation. It therefore plays an irrelevant role for flat extrinsic geometry. The expansions (3.10) are then inserted into the field equations that arise from the action (2.4) with the stress-energy tensor for the energy/matter fields given in (2.7). The resulting equations are regarded as "ultralocal" in the worldvolume coordinates and are as such simply linear ordinary differential equations for radial fluctuations of the metric, dilaton, and gauge field in the background (3.7).

It is interesting to note that the first order source terms appearing in the ODE's for the metric do not involve derivatives of the background scalar $\varphi$. The first order metric corrections $f_{\mu \nu}$ are therefore not altered by the non-trivial background to first order. In fact, they were determined in [12]. The constraint equations constitute a subset of the field equations, coming from the $(r b)$ component of the metric EOM and the $\left(a_{1} \ldots a_{p}\right)$ component of the gauge field EOM. To first order they take the form

$$
\nabla_{a} T^{a b}=j_{\phi} \partial^{b} \varphi, \quad \nabla_{a} J_{p+1}^{a a_{1} \ldots a_{p}}=0
$$

where we have introduced the modified current and dilaton current

$$
J_{p+1}=\star_{(p+1)}\left(e^{a_{p} \varphi / 2} Q_{p}\right), \quad j_{\phi}=\frac{a_{p}}{2} \Phi_{p} Q_{p} .
$$

The stress-energy tensor $T^{a b}$, the total electric charge $Q_{p}$ and electric potential $\Phi_{p}$ are given in appendix C.1. The equations (3.12) are valid for all values of $r$ up to first order in derivatives. One could now proceed order-by-order in the expansion, but since we are interested in simultaneously considering extrinsic perturbations of the worldvolume geometry we truncate at first order.

Extrinsic equations. We now repeat the same steps as in the analysis for the extrinsic perturbations performed for neutral branes ${ }^{8}$ in [22], but with a non-trivial background. To consider the extrinsic perturbations it is useful to introduce Fermi normal coordinates adapted to the worldvolume $\mathcal{W}_{p+1}$. In these coordinates, the metric is parametrized by the coordinates $\left(\sigma^{a}, y_{i}\right)$ where $y_{i}=0$ denotes the position of the worldvolume $\mathcal{W}_{p+1}$ in the transverse $(n+2)$-dimensional space. Thus we have that $\delta X^{i}(\sigma, y)=y^{i}$ for the transverse scalars around the flat embedding $\eta_{a b}$ and under the variation $X^{i} \rightarrow X^{i}+\delta X^{i}$ one finds that the induced metric transforms as $\delta \gamma_{a b}=-2 K_{a b}{ }^{i} \delta X_{i}=-2 K_{a b}{ }^{i} y_{i}$.

The perturbations along any of the coordinates $y_{i}$ decouple [49] and therefore we restrict the analysis to a given $i=\hat{i}$. Introducing the direction cosine $y_{\hat{i}}=r \cos \theta$ and noting that $r^{2}=y_{i} y^{i}$, a curved worldvolume metric $\gamma_{a b}$ to first order in derivatives in these coordinates can then be expanded as

$$
\gamma_{a b}=\eta_{a b}-2 K_{a b}{ }^{\hat{i}} r \cos \theta+\mathcal{O}\left(\tilde{\varepsilon}^{2}\right)
$$

\footnotetext{
${ }^{8}$ As noted in [22] the analysis of that paper still holds for charged branes and/or backgrounds with a non-trivial metric. The generalization below thus pertains mainly the fact that we allow for further non-trivial background fields.
} 
where $K_{a b} \hat{i}$ is a one-derivative term of $\mathcal{O}(\tilde{\varepsilon})$. This means that the leading order background metric (3.5) in the overlap region is given by

$$
\begin{aligned}
\mathrm{d} s^{2} & =\left(\eta_{a b}-2 K_{a b}{ }^{\hat{i}} r \cos \theta\right) \mathrm{d} \sigma^{a} \mathrm{~d} \sigma^{b}+\mathrm{d} r^{2}+r^{2}\left(\mathrm{~d} \theta^{2}+\sin ^{2} \theta \mathrm{d} \Omega_{(n)}^{2}\right), \\
C_{p+1} & =\mathcal{C}_{p+1}(y), \quad \phi=\varphi(y) .
\end{aligned}
$$

Note that in this case we only allow the background fields to have dependence on the transverse coordinates $y$, so that the extrinsic perturbations are decoupled from the intrinsic ones. We also remark that we can assume that the background field strength is non-zero only in the singled-out direction $y^{\hat{i}}$. In principle, $\mathcal{F}_{p+2}=\mathrm{d} \mathcal{C}_{p+1}$ could have non-zero components in two or more transverse directions, however, it is not difficult to realize that such components do not play a role to leading order and only become important at higher orders.

We now consider a dipole-type perturbation to first order in $\tilde{\varepsilon}$ and write the expansion of the fields as

$$
\mathrm{d} s^{2}=\mathrm{d} s_{(0)}^{2}+\tilde{\varepsilon} \cos \theta d_{\mu \nu}(r) \mathrm{d} x^{\mu} \mathrm{d} x^{\nu}, \quad \phi=\phi^{(0)}+\tilde{\varepsilon} \phi^{(1)}, \quad F_{p+2}=\mathrm{d} C_{p+1}^{(0)}+\tilde{\varepsilon} \mathrm{d} C_{p+1}^{(1)},
$$

where we regard (3.7) as the first term in the expansion (denoted by subscript (0)), and we note that $\mathrm{d} y^{\hat{i}}=(\cos \theta \mathrm{d} r-r \sin \theta \mathrm{d} \theta)$. Analogous to [22] we focus on the large $r$ region of the near-zone solution. In this region, the metric takes the form

$$
\begin{aligned}
\mathrm{d} s^{2}= & \left(\eta_{a b}-2 K_{a b}{ }^{\hat{i}} r \cos \theta+\frac{16 \pi G}{n \Omega_{(n+1)}}\left(T_{a b}(\sigma)-\frac{T(\sigma)}{D-2} \eta_{a b}\right) \frac{1}{r^{n}}\right) \mathrm{d} \sigma^{a} \mathrm{~d} \sigma^{b} \\
& +\left(1-\frac{16 \pi G}{\Omega_{(n+1)}} \frac{1}{D-2} \frac{T(\sigma)}{r^{n}}\right) \mathrm{d} r^{2}+r^{2}\left(\mathrm{~d} \theta^{2}+\sin ^{2} \theta \mathrm{d} \Omega_{(n)}^{2}\right) \\
& +\tilde{\varepsilon} \cos \theta d_{\mu \nu}(r) \mathrm{d} x^{\mu} \mathrm{d} x^{\nu}+\mathcal{O}\left(\tilde{\varepsilon}^{2}\right)+\mathcal{O}\left(T_{a b}^{2} / r^{2 n}\right),
\end{aligned}
$$

while the field strength and dilaton are given by

$$
\begin{aligned}
F_{p+2} & =F_{p+2}^{(\mathrm{M})}+\tilde{\varepsilon} \mathcal{F}_{p+2}+\mathcal{O}\left(\tilde{\varepsilon}^{2}\right)+\mathcal{O}\left(T_{a b}^{2} / r^{2 n}\right), \\
\mathrm{d} \phi & =\mathrm{d} \phi^{(\mathrm{M})}+\tilde{\varepsilon} \mathrm{d} \varphi+\mathcal{O}\left(\tilde{\varepsilon}^{2}\right)+\mathcal{O}\left(T_{a b}^{2} / r^{2 n}\right) .
\end{aligned}
$$

The first order corrections $\mathrm{d} C_{p+1}^{(1)}$ and $\mathrm{d} \phi^{(1)}$ drop out as they appear at higher order in the large $r$ expansion. This also means that only the monopole part - by definition of order $\mathcal{O}\left(T_{a b} / r^{n}\right)$ - of $\mathrm{d} C_{p+1}^{(0)}$ and $\mathrm{d} \phi^{(0)}$ plays a role, which we denote by $F_{p+2}^{(\mathrm{M})}$ and $\mathrm{d} \phi^{(\mathrm{M})}$ and are given by

$$
F_{p+2}^{(M)}=-e^{-a_{p} \varphi / 2} \frac{16 \pi G}{\Omega_{(n+1)}} \frac{Q_{p}}{r^{n+1}} \mathrm{~d} r \wedge \star_{(p+1)} 1, \quad \mathrm{~d} \phi^{(M)}=-\frac{16 \pi G}{\Omega_{(n+1)}} \frac{a_{p} \Phi_{p} Q_{p}}{2 r^{n+1}} \mathrm{~d} r .
$$

and are thus determined by the current source (3.13) of the brane.

In the presence of non-trivial background fields, we therefore see that the bulk stress tensor $\mathbf{T}_{\mathbf{M} \mu \nu}$ does not vanish at large $r$ distances. Indeed, from (3.18) the stress-energy 
tensor consists of a simple pole-dipole term and in particular does not involve the metric and background field perturbations. The combination

$$
(n+1) \csc \theta\left(G_{r \theta}-8 \pi G \mathbf{T}_{\mathbf{M} r \theta}\right)-r \sec \theta\left(G_{r r}-8 \pi G \mathbf{T}_{\mathbf{M} r r}\right)=0,
$$

is therefore still a constraint equation ${ }^{9}$ with $\mathbf{T}_{\mathbf{M} \mu \nu}=\mathbf{T}_{\mathbf{M} \mu \nu}^{(F)}+\mathbf{T}_{\mathbf{M} \mu \nu}^{(\phi)}$ and takes the modified form

$$
\frac{n+2}{r^{n}} \frac{8 \pi G}{\Omega_{(n+1)}}\left(T^{a b} K_{a b} \hat{i}-\mathcal{F}^{\hat{i}}\right)=0
$$

where $\mathcal{F}^{\hat{i}}$ is the induced force term given by the pole-dipole interaction term

$$
\mathcal{F}^{\hat{i}}=\frac{\Omega_{(n+1)}}{n+2} r^{n}\left((n+1) \csc \theta \mathbf{T}_{\mathbf{M} r \theta}-r \sec \theta \mathbf{T}_{\mathbf{M} r r}\right) .
$$

To evaluate the force term (3.22) we use the expressions (2.7) for the stress-energy tensor, insert the expansion (3.18) keeping only the pole-dipole contribution and then use (3.19) along with (3.13). Covariantizing the result for any $i=\hat{i}$, we arrive at the following constraint equation

$$
T^{a b} K_{a b}^{i}=n_{\mu}^{i}\left(\frac{1}{(p+1) !} \mathcal{F}_{p+2}^{\mu \mu_{1} \ldots \mu_{p+1}} J_{p+1 \mu_{1} \ldots \mu_{p+1}}+j_{\phi} \partial^{\mu} \varphi\right)
$$

where the currents $J_{p+1}$ and $j_{\phi}$ are given by eq. (3.13). We emphasize that the quantities appearing in the constraint equation (3.23) are evaluated in the region $r_{H} \ll r \ll \min (\mathcal{R}, L)$. Together (3.12) and (3.23) are in agreement with eqs. (2.12)-(2.14) for a localized stressenergy tensor and current.

\subsection{Constraint equations for the $\mathrm{D} p$-F1 bound state}

We now apply the same procedure to the non-extremal $\mathrm{D} p$-F1 bound state, which will exhibit some new features. The non-extremal D $p$-F1 bound state in the Einstein frame has the metric [50]

$$
\begin{aligned}
\mathrm{d} s^{2}= & D^{\frac{1-p}{8}} H^{\frac{p-7}{8}}\left(-f u_{a} u_{b}+v_{a} v_{b}\right) \mathrm{d} \sigma^{a} \mathrm{~d} \sigma^{b}+D^{\frac{9-p}{8}} H^{\frac{p-7}{8}} \perp_{a b} \mathrm{~d} \sigma^{a} \mathrm{~d} \sigma^{b} \\
& +D^{\frac{1-p}{8}} H^{\frac{p+1}{8}}\left(f^{-1} \mathrm{~d} r^{2}+r^{2} \mathrm{~d} \Omega_{8-p}^{2}\right)
\end{aligned}
$$

where $u_{a}$ is a normalized timelike vector (the boost velocity), $v_{a}$ is a normalized spatial vector characterizing the direction of the F1-string satisfying the orthogonality condition $u^{a} v_{a}=0$ and the projector onto the worldvolume directions orthogonal to the string is $\perp_{b}^{a} \equiv \delta^{a}{ }_{b}+u^{a} u_{b}-v^{a} v_{b}$. The dilaton is given by

$$
e^{2(\phi-\varphi)}=D^{\frac{p-5}{2}} H^{\frac{3-p}{2}},
$$

and the gauge fields are ${ }^{10}$

$$
\begin{aligned}
B_{2} & =e^{-a_{\mathrm{F} 1} \varphi / 2} \sin \xi\left(H^{-1}-1\right) \operatorname{coth} \alpha u \wedge v, \\
C_{p-1} & =(-1)^{p} e^{-a_{p-2} \varphi / 2} \tan \xi\left(D H^{-1}-1\right) \star_{(p+1)}(u \wedge v), \\
C_{p+1} & =(-1)^{p} e^{-a_{p} \varphi / 2} \cos \xi\left(H^{-1}-1\right) \operatorname{coth} \alpha \star_{(p+1)} \mathbf{1},
\end{aligned}
$$

\footnotetext{
${ }^{9}$ When no background fields are present $\left(\mathbf{T}_{\mathbf{M} \mu \nu}=0\right)$, the constraint equation (3.20) was obtained in [22].

${ }^{10}$ Beware of the solution in ref. [50] which has a typo in the $C_{p+1}$ field that has been correct in ref. [33] for the D3-F1 solution.
} 
where $B_{2}$ is the NSNS two-form, and $C_{p-1}$ and $C_{p+1}$ are the $(p-1)$-form and $(p+1)$-form RR fields, respectively. The dilaton coupling constants are $a_{q}=(3-q) / 2$ and $a_{\mathrm{F} 1}=-1$. The Hodge star operator $\star_{(p+1)}$ is defined with respect to the $(p+1)$-dimensional worldvolume metric. The structure functions $f \equiv f(r), D \equiv D(r)$ and $H \equiv H(r)$ are

$$
f(r)=1-\frac{r_{0}^{n}}{r^{n}}, \quad H(r)=1+\frac{r_{0}^{n}}{r^{n}} \sinh ^{2} \alpha, \quad D^{-1}(r)=\cos ^{2} \xi+H^{-1} \sin ^{2} \xi,
$$

with $n=7-p$. The solution depends on three real parameters; $r_{0}>0, \alpha$, and the angle $\xi \in[0,2 \pi[$. For $\xi=0$, the solution reduces to the $p$-brane solution (3.1) in ten dimensions. Following the previous subsection, we have already included in the expressions above the redundant shift $\varphi$ of the dilaton to make this particular symmetry of the action manifest. The field strengths are $\mathrm{ar}^{11}$

$$
H_{3}=\mathrm{d} B_{2}, \quad F_{p}=\mathrm{d} C_{p-1}, \quad \tilde{F}_{p+2}=\mathrm{d} C_{p+1}-H_{3} \wedge C_{p-1} .
$$

For the $\tilde{F}_{p+2}$ to be invariant under the gauge transformation $\delta C_{p-1}=\mathrm{d} \Lambda_{p-2}$, the $(p+1)$ form potential should transform as $\delta C_{p+1}=\mathrm{d} \Lambda_{p-2} \wedge B_{2}$. The $(p+2)$-form is invariant under the gauge transformation $\delta C_{p+1}=\mathrm{d} \Lambda_{p}$. The effective currents and charges are given in appendix C.

Ansatz and background fields. The perturbation ansatz is constructed from the bound-state solution (3.24)-(3.30) by promoting the parameters $u^{a}, v^{a}, r_{0}, \alpha, \xi$ to functions of the worldvolume coordinates $\sigma^{a}$, as well as promoting the worldvolume metric $\eta_{a b} \rightarrow \gamma_{a b}(\sigma)$. In addition, we now include the general background gauge fields $\mathcal{B}_{2}, \mathcal{C}_{p-1}$, $\mathcal{C}_{p+1}$ and dilaton $\varphi$ in the overlap region (cf. (3.6)). Explicitly, they enter through the associated field strengths

$$
\mathcal{H}_{3}=\mathrm{d} \mathcal{B}_{2}, \quad \mathcal{F}_{p}=\mathrm{d} \mathcal{C}_{p-1}, \quad \tilde{\mathcal{F}}_{p+2}=\mathrm{d} \mathcal{C}_{p+1}-\mathcal{H}_{3} \wedge \mathcal{C}_{p-1} .
$$

In analogy with (3.7), the ansätze for the field strengths are thus composed by taking

$$
H_{3} \rightarrow H_{3}+\mathcal{H}_{3}, \quad F_{p} \rightarrow F_{p}+\mathcal{F}_{p} \quad \text { and } \quad \tilde{F}_{p+2} \rightarrow \tilde{F}_{p+2}+\tilde{\mathcal{F}}_{p+2} .
$$

In this way, the background fields do not affect the solution at leading order in the derivative expansion and the ansatz asymptotes at large $r$ to the pull-back of the background fields

$$
H_{3}=\mathcal{H}_{3}(\sigma, y), \quad F_{p}=\mathcal{F}_{p}(\sigma, y), \quad \tilde{F}_{p+2}=\tilde{\mathcal{F}}_{p+2}(\sigma, y), \quad \phi=\varphi(\sigma, y) .
$$

The asymptotic charges are altered accordingly

$$
\mathbf{Q}_{\mathrm{F} 1}=e^{a_{\mathrm{F} 1} \varphi / 2} \mathcal{Q}_{\mathrm{F} 1}+(-1)^{p} e^{a_{p} \varphi / 2} Q_{p} \mathcal{C}_{p-1}, \quad \mathbf{Q}_{p-2}=e^{a_{p-2} \varphi / 2} \mathcal{Q}_{p-2}, \quad \mathbf{Q}_{p}=e^{a_{p} \varphi / 2} Q_{p},
$$

where the charge densities $\mathcal{Q}_{\mathrm{F} 1}, \mathcal{Q}_{p-2}$ and total charge $Q_{p}$ are given by eq. (C.11) and the currents take the expected form

$$
j_{2}=\mathbf{Q}_{\mathrm{F} 1} u \wedge v, \quad J_{p-1}=\mathbf{Q}_{p-2}{ }_{(p+1)}(u \wedge v), \quad J_{p+1}=\mathbf{Q}_{p} \star_{(p+1)} \mathbf{1} .
$$

In the following we consider the intrinsic and extrinsic perturbations separately.

\footnotetext{
${ }^{11}$ For $p=3$, the composite five-form field strength $\tilde{F}_{5}$ should be made self-dual.
} 
Intrinsic equations. We restrict the dependence of the background fields to the worldvolume coordinates $\sigma^{a}$ and consider each field in a derivative expansion analogous to eq. (3.10) allowing in principle for the construction of a multi-charged bound state solution order-by-order. We note that since the fields are restricted to worldvolume coordinate dependence only, the background field $\mathcal{C}_{p+1}$ simply corresponds to a pure gauge transformation analogous to section 3.1.

We are interested in the subset of field equations eqs. (B.2) (and (B.6)) that constitute the set of constraint equations. Taking particular combinations of the constraint equations, which we list in appendix C.4, these can be expressed as stress-energy and current conservation equations on the worldvolume

$$
\begin{aligned}
\nabla_{a} T^{a b}= & \frac{1}{(p-1) !}\left[\mathcal{F}_{p}^{b a_{1} \ldots a_{p-1}} J_{p-1 a_{1} \ldots a_{p-1}}+(-1)^{p+1} \frac{1}{2} \mathcal{H}_{3}^{b a_{1} a_{2}} \mathcal{C}_{p-1}^{a_{3} \ldots a_{p+1}} J_{p+1 a_{1} \ldots a_{p+1}}\right] \\
& +\frac{1}{2} \mathcal{H}_{3}^{b a_{1} a_{2}} j_{2 a_{1} a_{2}}+j_{\phi} \partial^{b} \varphi \\
\nabla_{a} j_{2}^{a b}= & 0, \quad \nabla_{a} J_{p-1}^{a b_{1} \ldots b_{p-2}}=\frac{1}{3 !} \mathcal{H}_{3 a b c} J_{p+1}^{a b c b_{1} \ldots b_{p-2}}, \quad \nabla_{a} J_{p+1}^{a b_{1} \ldots b_{p}}=0,
\end{aligned}
$$

where the dilaton current is related to the charges such that

$$
j_{\phi}=\frac{1}{2}\left(a_{\mathrm{F} 1} \mathcal{Q}_{\mathrm{F} 1} \Phi_{\mathrm{F} 1}+a_{p} Q_{p} \Phi_{p}\right) .
$$

These are worldvolume equations and are satisfied for all values of $r$ up to first order in derivatives. The stress-energy tensor is given in eq. (C.8) and the currents in eq. (3.35).

Extrinsic equations. For the extrinsic perturbations we restrict the dependence of the background fields to the transverse space spanned by the coordinates $y^{i}$ and consider the perturbation to first order in a single direction $y^{\hat{i}}=r \cos \theta$. The $r$-asymptotics of the near-zone field strengths therefore follow a similar form as (3.18), explicitly

$$
\begin{array}{cc}
H_{3}=H_{3}^{(M)}+\tilde{\varepsilon} \mathcal{H}_{3}+\mathcal{O}\left(\tilde{\varepsilon}^{2}\right)+\mathcal{O}\left(T_{a b}^{2} / r^{2 n}\right), & F_{p}=F_{p}^{(M)}+\tilde{\varepsilon} \mathcal{F}_{p}+\mathcal{O}\left(\tilde{\varepsilon}^{2}\right)+\mathcal{O}\left(T_{a b}^{2} / r^{2 n}\right), \\
\tilde{F}_{p+2}=\tilde{F}_{p+2}^{(M)}+\tilde{\varepsilon} \tilde{\mathcal{F}}_{p+2}+\mathcal{O}\left(\tilde{\varepsilon}^{2}\right)+\mathcal{O}\left(T_{a b}^{2} / r^{2 n}\right), & \mathrm{d} \phi=\mathrm{d} \phi^{(\mathrm{M})}+\tilde{\varepsilon} \mathrm{d} \varphi+\mathcal{O}\left(\tilde{\varepsilon}^{2}\right)+\mathcal{O}\left(T_{a b}^{2} / r^{2 n}\right),
\end{array}
$$

while the asymptotic metric is again of the form given by eq. (3.17). The monopole parts of the leading order fields are

$$
\begin{aligned}
& H_{3}^{(M)}=-e^{-a_{\mathrm{F} 1} \varphi / 2} \frac{16 \pi G}{\Omega_{(n+1)}} \frac{\mathcal{Q}_{\mathrm{F} 1}}{r^{n+1}} \mathrm{~d} r \wedge u \wedge v, \\
& F_{p}^{(M)}=(-1)^{p+1} e^{-a_{p-2} \varphi / 2} \frac{16 \pi G}{\Omega_{(n+1)}} \frac{\mathcal{Q}_{p-2}}{r^{n+1}} \mathrm{~d} r \wedge \star_{(p+1)}(u \wedge v), \\
& \tilde{F}_{p+2}^{(M)}=(-1)^{p+1} e^{-a_{p} \varphi / 2} \frac{16 \pi G}{\Omega_{(n+1)}} \frac{Q_{p}}{r^{n+1}} \mathrm{~d} r \wedge \star_{(p+1)} \mathbf{1}, \quad \mathrm{d} \phi^{(M)}=-\frac{16 \pi G}{\Omega_{(n+1)}} \frac{j_{\phi}}{r^{n+1}} \mathrm{~d} r,
\end{aligned}
$$

where $j_{\phi}$ is given by eq. (3.37).

The constraint equations can again be extracted through the combination of the bulk stress-energy tensor given by eq. (3.20), where the force terms arising from the presence of 
the background fields are given by eq. (3.22). In particular, the individual covariantized contributions are

$$
\begin{aligned}
\mathcal{F}_{H}^{\mu} & =\frac{1}{2} \mathcal{H}_{3}^{\mu \mu_{1} \mu_{2}}\left(j_{2 \mu_{1} \mu_{2}}+(-1)^{p+1} \frac{1}{(p-1) !} \mathcal{C}_{p-1}^{\mu_{3} \ldots \mu_{p+1}} J_{p+1 \mu_{1} \ldots \mu_{p+1}}\right), \\
\mathcal{F}_{F_{p}}^{\mu} & =\frac{1}{(p-1) !} \mathcal{F}_{p}^{\mu \mu_{1} \ldots \mu_{p-1}} J_{p-1 \mu_{1} \ldots \mu_{p-1}}, \\
\mathcal{F}_{\tilde{F}_{p+2}}^{\mu} & =\frac{1}{(p+1) !} \tilde{\mathcal{F}}_{p+2}^{\mu \mu_{1} \ldots \mu_{p+1}} J_{p+1 \mu_{1} \ldots \mu_{p+1}}, \quad \mathcal{F}_{\phi}^{\mu}=j_{\phi} \partial^{\mu} \varphi .
\end{aligned}
$$

We note that the currents $j_{2}, J_{p-1}$ and $J_{p+1}$ are given by eq. (3.35) in terms of the modified charges. The extrinsic equations thus take the form

$$
T^{a b} K_{a b}^{i}=n_{\mu}^{i}\left(\mathcal{F}_{H}^{\mu}+\mathcal{F}_{F_{p}}^{\mu}+\mathcal{F}_{F_{p+2}}^{\mu}+\mathcal{F}_{\phi}^{\mu}\right)
$$

This is exactly the stress-energy tensor conservation equation in the perpendicular directions to the worldvolume.

Finally, we note that the intrinsic equations (3.36) together with the extrinsic equations (3.41) are in agreement with the far-region analysis (2.22) for localized stress-energy tensor and currents (see appendix B) once we replace in those expressions the fields with their pull-back onto the worldvolume (analogous to the discussion at the end of section 2.1), i.e. $\tilde{F}_{q} \rightarrow \tilde{\mathcal{F}}_{q}$ for all $q$ and also $\phi \rightarrow \varphi$. Notice that in this procedure it is understood that both the fields and their derivatives are pulled-back onto the worldvolume, so e.g. $\partial_{i} \varphi=\left.\left[\partial_{i} \phi\left(X^{\mu}(\sigma, y)\right)\right]\right|_{y=0}$.

It was shown in [30] that the combined set of equations (3.36), (3.41) in a trivial flat space background at extremality are equivalent to the equations of motion of the $(p+1)$ dimensional DBI action with an electric field constraint (see eq. (7.34) in [30]). It would be interesting to extend the analysis of [30] to non-trivial backgrounds using the general form of (3.36), (3.41) derived here.

\section{External couplings from hydrostatic partition functions}

In this section we consider another method by which one can derive the couplings to backgrounds fields and their consequences in the form of the equations of motion. This method relies on obtaining the hydrostatic partition function from the Euclidean on-shell action for black holes and requires local measurements of temperature and chemical potentials as well as the measurement of stress-energy, electric and dilaton currents. As is well-known, the existence of a hydrostatic partition function requires the existence of a timelike Killing vector field along which the fluid velocity is aligned [7]. ${ }^{12}$ Therefore, throughout this section we focus on fluids in stationary equilibrium. Nevertheless, the final form of the equations of motion that arise from the partition function, including force terms, is completely general. We begin with general considerations on gravitational partition functions and then derive

\footnotetext{
${ }^{12}$ It may be possible to relax the requirement of stationarity or, more generally, of non-dissipative flows, as advertised in [51, 52], by doubling the number of degrees of freedom.
} 
the external couplings for black $p$-branes carrying Maxwell charge as well as for black $p$ branes charged under a $p$-form gauge field. The method can be applied to any bound state such as the $\mathrm{D} p$-F1 analyzed in the previous section and we leave this longer endeavor for future work.

\subsection{General considerations on partition functions}

In a semi-classical approximation, the partition function $\mathcal{Z}$ for a given black hole solution can be obtained by evaluating the Euclidean on-shell action $I_{\mathrm{E}}$ for that particular solution such that [53]

$$
\ln \mathcal{Z}=i I_{\mathrm{E}}
$$

where we have Wick rotated the time coordinate $t \rightarrow i t$. In this approximation, the Euclidean action yields the Gibbs free energy of the black hole, which takes the generic form

$$
i I_{\mathrm{E}}=\beta\left(M-T S-\Omega J-\Phi_{\mathrm{H}} Q_{p}\right),
$$

where $\beta=T^{-1}$ is the radius of the time circle, $T$ is the global temperature, $M$ the total energy, $\Omega$ the angular velocity, $J$ the angular momentum, $\Phi_{\mathrm{H}}$ the global chemical potential and $Q_{p}$ the total electric charge of the black hole. The generic form of (4.2) is a consequence of stationarity. Therefore, the partition function obeys the relation

$$
d(T \ln \mathcal{Z})=S d T+J d \Omega+Q_{p} d \Phi_{\mathrm{H}},
$$

consistent with the first law of thermodynamics, which leads to the following thermodynamic identities

$$
S=\frac{\partial(T \ln \mathcal{Z})}{\partial T}, \quad J=\frac{\partial(T \ln \mathcal{Z})}{\partial \Omega}, \quad Q_{p}=\frac{\partial(T \ln \mathcal{Z})}{\partial \Phi_{\mathrm{H}}} .
$$

In the following, we will consider the partition function of a generic black $p$-brane with a definite temperature, chemical potential (or equivalently horizon radius $r_{0}$ and charge parameter $\alpha$ ), boost velocity $u^{a}$ and boundary values of the external gauge fields $\mathcal{C}_{q+1}$ and dilaton field $\varphi$. Furthermore, due to stationarity, we assume that the boost velocity is aligned with a worldvolume Killing vector field $\mathbf{k}^{a}$ such that $u^{a}=\mathbf{k}^{a} / \mathbf{k}$ with $\mathbf{k}$ being its norm. Due to the translational invariance of the partition function along the worldvolume $\mathcal{W}_{p+1}$, the partition function must factorize,

$$
\mathcal{Z}=\prod_{\mathcal{W}_{p+1}} \mathcal{Z}_{V_{p}}
$$

where $\mathcal{Z}_{V_{p}}$ denotes the partition function of the arbitrarily small $p$-dimensional volume. We now consider perturbing the black brane to a new stationary solution by allowing the black brane parameters to depend on the worldvolume coordinates $\sigma^{a}$. In this case, the partition function takes the form

$$
\mathcal{Z}=\prod_{\mathcal{W}_{p+1}} \mathcal{Z}_{V_{p}}(\sigma)+\mathcal{O}(\varepsilon, \tilde{\varepsilon}),
$$


where the derivative corrections of order $\mathcal{O}(\varepsilon, \tilde{\varepsilon})$ are evaluated from solving the equations of motion for a given theory and determining the corresponding perturbations. However, in order to derive the constraint equations obtained in the previous sections, it is not necessary to consider higher-order corrections. Therefore, in what follows we will restrict the analysis to the leading order case for which the partition function of the leading order solution takes the form

$$
\ln \mathcal{Z}=\int_{\mathcal{W}_{p+1}}{ }_{(p+1)} \ln \mathcal{Z}_{0}\left[\gamma_{a b}, \mathbf{k}^{b}, X^{i}, \mathcal{C}_{q+1}, \varphi\right],
$$

where $\ln \mathcal{Z}_{0}$ denotes the partition function of the uncorrected $p$-brane as a function of the external background sources. Here, the worldvolume Killing vector field $\mathbf{k}^{a}$ should be understood as the pull-back of a background Killing vector field $\mathbf{k}^{\mu}$ and $X^{i}$ the transverse embedding scalars denoting the position of the worldvolume in the ambient space. The external gauge field and dilaton in (4.7) should be understood either as the pull-back onto the worldvolume of the external fields or as the components of the external fields projected and evaluated on the worldvolume.

Except for possible gauge or gravitational anomalies, the partition function (4.7) must be invariant under diffeomorphisms and gauge transformations. Under worldvolume diffeomorphisms the induced metric transforms as $\delta_{\|} \gamma_{a b}=2 \nabla_{(a} \xi_{b)}$, the gauge field transform as $\delta_{\|} \mathcal{C}_{q+1}=\xi^{a} \nabla_{a} \mathcal{C}_{q+1 \mu_{1} \ldots \mu_{q+1}}+\nabla_{\mu_{1}} \xi^{\nu} \mathcal{C}_{q+1 \nu \mu_{2} \ldots \mu_{q+1}}+\ldots+\nabla_{\mu_{q+1}} \xi^{\nu} \mathcal{C}_{q+1 \mu_{1} \mu_{2} \ldots \nu}$ and the dilaton transforms as $\delta_{\|} \varphi=\xi^{a} \partial_{a} \varphi$. The Killing vector is held fixed, due to stationarity, as well as the transverse scalars since we are performing a Lagrangian type variation. ${ }^{13}$ Assuming that no boundaries are present, this leads to the variation of the partition function



where we have used the fact that invariance of (4.7) under gauge transformations $\delta \mathcal{C}_{q+1}=$ $d \Lambda_{q}$ leads to the current conservation equation

$$
\nabla_{a_{1}} J_{q+1}^{a_{1} \ldots a_{q+1}}=0
$$

In (4.8) we have introduced the worldvolume stress-energy tensor, electric current and dilaton current via the expressions

$$
T^{a b}=-\frac{2}{\beta \sqrt{-\gamma}} \frac{\delta \ln \mathcal{Z}}{\delta \gamma_{a b}}, \quad J_{q+1}^{a_{1} \ldots a_{q+1}}=-\frac{1}{\beta \sqrt{-\gamma}} \frac{\delta \ln \mathcal{Z}}{\delta \mathcal{C}_{q+1 a_{1} \ldots a_{q+1}}}, \quad j_{\phi}=-\frac{1}{\beta \sqrt{-\gamma}} \frac{\delta \ln \mathcal{Z}}{\delta \varphi} .
$$

Since (4.8) must hold for arbitrary $\xi_{b}$ we find that we must have

$$
\nabla_{a} T^{a b}=\frac{1}{(q+1) !} \mathcal{F}_{q+2 a_{1} \ldots a_{q+1}}^{b} J_{q+1}^{a_{1} \ldots a_{q+1}}+j_{\phi} \partial^{b} \varphi
$$

\footnotetext{
${ }^{13}$ Lagrangian type variations are variations in which the worldvolume position, characterized by the transverse scalars $X^{i}$, is held fixed and the background metric is displaced. Alternatively, one may consider a variational scheme in which the background metric is held fixed and the transverse scalars are displaced. These two types of variations are equivalent, even to higher order in derivatives [54].
} 
Analogously, considering a diffeomorphism in the directions orthogonal to the worldvolume the induced metric transforms as $\delta_{\perp} \gamma_{a b}=-2 K_{a b}{ }^{i} \xi_{i}$ and one finds the equation of motion

$$
T^{a b} K_{a b}^{i}=\frac{1}{(q+1) !} \mathcal{F}_{q+2 a_{1} \ldots a_{q+1}}^{i} J_{q+1}^{a_{1} \ldots a_{q+1}}+j_{\phi} \partial^{i} \varphi .
$$

Once the partition function (4.7) is given in terms of the background sources, one may just use (4.10) to obtain the stress-energy tensor and currents while under direct variation with respect to $X^{i}$ one obtains the equation of motion (4.12) and hence the non-trivial form of the force terms.

It is interesting to note that the partition function (4.7) can be written as a localized integral over the spacetime, i.e.

$$
\ln \mathcal{Z}=\int_{\mathcal{M}_{D}} \star \ln \mathcal{Z}_{0}\left[\gamma_{a b}, \mathbf{k}^{b}, X^{i}, \mathcal{C}_{q+1}, \varphi\right] \tilde{\delta}^{(n+2)}\left(x^{i}-X^{i}\right)
$$

where $x^{i}$ are spacetime coordinates and $\tilde{\delta}^{(n+2)}$ the reparametrization invariant delta function in the transverse $(n+2)$-dimensional space. Written in this form, one can extract the spacetime stress tensor, which takes the general form

$$
\mathbf{T}^{\mu \nu}=-\frac{2}{\sqrt{-g}} \frac{\delta(T \ln \mathcal{Z})}{\delta g_{\mu \nu}}=T^{a b} \partial_{a} X^{\mu} \partial_{b} X^{\nu} \tilde{\delta}^{(D-p-1)}\left(x^{i}-X^{i}\right),
$$

where $T^{a b}$ is the worldvolume stress-energy tensor obtained using (4.10) and $\partial_{a} X^{\mu}$ is a projector along the worldvolume directions. Here, $X^{\mu}$ is the set of mapping functions which includes the $(p+1)$-worldvolume directions besides the transverse scalars $X^{i}$. Therefore, to leading order, spacetime stress-energy tensors that arise from (4.7) represent localized objects in the ambient background with metric $g_{\mu \nu}$.

We note that even though stationarity will impose some restrictions on the form of the currents or configurations that solve (4.11)-(4.12), the form of the equations of motion (4.11)-(4.12), obtained by demanding diffeomorphism and gauge invariance of the partition function, is completely general and matches exactly those obtained in (2.12) and (2.13) derived from (2.9) for a stress-energy tensor of the form (4.14) as well as with localized electric and dilaton currents and without a magnetic current.

\subsection{External couplings for black branes carrying Maxwell charge}

In this section we consider the case of black $p$-branes carrying Maxwell charge, which were not analyzed in the previous sections. These are also solutions to the action (2.4) but with a two form field strength $F_{2}(q=0)$ and for simplicity we consider the case where no dilaton field is present. The metric and gauge field can be found in [11] and read

$$
\begin{aligned}
d s^{2} & =H^{N-2}\left(\left(P_{a b}-H^{-N} f u_{a} u_{b}\right) \mathrm{d} \sigma^{a} \mathrm{~d} \sigma^{b}+f^{-1} d r^{2}+r^{2} \mathrm{~d} \Omega_{(n+1)}^{2}\right), \\
C_{1} & =\frac{\sqrt{N}}{H}\left(\frac{r_{0}}{r}\right)^{n} \sinh \alpha \cosh \alpha u_{a} \mathrm{~d} \sigma^{a}, \quad N=\frac{2(n+p+1)}{n+p},
\end{aligned}
$$

where the functions $f$ and $H$ were given in (3.2). Here $r_{0}$ and $\alpha$ are the horizon radius and charge parameter, respectively. In order to evaluate the Euclidean on-shell action we 
need to add appropriate boundary counter-terms to the action (2.4). Using the fact that on-shell (for general $q$ ) one has

$$
\star R=\frac{1}{2} d \phi \wedge \star d \phi+e^{a_{q} \phi}\left(\frac{1}{2}-\frac{q+1}{D-2}\right) F_{q+2} \wedge \star F_{q+2},
$$

the Euclidean action over a $D$-dimensional region $Y$ with boundary $\partial Y$ for $q=0$ and $a_{q}=0$ becomes

$$
I_{\mathrm{E}}=\frac{1}{8 \pi G} \int_{\partial Y} \star[K]-\frac{1}{16 \pi G} \frac{1}{(D-2)} \int_{\partial Y} C_{1} \wedge \star F_{2},
$$

where the boundary $\partial Y$ is chosen to be a constant radial slice at infinity in the geometry of $(4.15)$ and where $[K]$ denotes the difference between the extrinsic curvature of a constant radial slice in (4.15) and the analogous radial slice in flat spacetime written in the same coordinates as in (4.15) but with an appropriate temperature redshift at infinity.

Analogous to the case considered in section 3.1 of $p$-branes with a top-form, we construct the leading order ansatz by adding a constant gauge field $\mathcal{C}_{1}$ to $C_{1}$ via a local gauge transformation such that, without loss of generality, the boosted gauge field (4.15) is given by

$$
C_{1} \rightarrow C_{1}+\mathcal{C}_{1 b} u^{b} u_{a} d \sigma^{a}
$$

However, such gauge transformation does not affect the evaluation of (4.17). This is because in order to evaluate the Euclidean action one must require the gauge field to be regular at the horizon by subtracting its value at the horizon as in [53]. In practice, this means that we must perform the shift $C_{1} \rightarrow C_{1}-\left(\Phi_{p}+\mathcal{C}_{1 b} u^{b}\right) u_{a} d \sigma^{a}$ via a local gauge transformation and therefore removing potential contributions due to $\mathcal{C}_{1}$. Given this, explicit evaluation of (4.17) leads to the Euclidean on-shell action

$$
I_{\mathrm{E}}=-i \beta \frac{\Omega_{(n+1)}}{16 \pi G} \int_{\mathcal{B}_{p}} \star_{(p)} r_{0}^{n},
$$

where we have taken the worldvolume geometry to be $\mathcal{W}_{p+1}=\mathbb{R} \times \mathcal{B}_{p}$ with $\mathcal{B}_{p}$ being the spatial part and $\star_{(p)} 1=\sqrt{-\gamma} d \sigma^{1} \wedge \ldots \wedge d \sigma^{p}$ (see appendix A). We note that (4.19) is simply proportional to the pressure $P$ as written explicitly in appendix C.2 and that it must be extremized at fixed global temperature and chemical potential.

The integrand in (4.19) is a local version of the Gibbs free energy of the brane. In order to obtain the partition function one must re-express it in terms of the background sources as in (4.7) for stationary configurations. In order to do so, we must demand gauge invariance, which from (4.9) implies that the electric current must be conserved $\nabla_{a} J_{1}^{a}=0$. Furthermore, we must also demand worldvolume diffeomorphism invariance which, in this case, using (4.11), requires that

$$
\nabla_{a} T^{a b}=\mathcal{F}_{2 a}^{b} J_{1}^{a}
$$

where both the stress-energy tensor and electric current are given in appendix C.2. The projection of this equation along the fluid flows $u_{a}$ is automatically satisfied due to the fact that the r.h.s. vanishes by symmetry while the l.h.s. vanishes due to the fact that the 
electric current is conserved and assuming the existence of a conserved entropy current $J_{s}^{a}=s u^{a}$. The projection of eq. (4.20) perpendicular to the fluid flows leads to

$$
\mathcal{T} s P_{b}^{c}\left(\partial^{b} \ln \mathcal{T}+a^{b}\right)+\mathcal{Q}\left(\Phi_{p}-\mathcal{C}_{1 a} u^{a}\right) P_{b}^{c}\left(\partial^{b} \ln \left(\Phi_{p}-\mathcal{C}_{1 a} u^{a}\right)+a^{b}\right)=0,
$$

where $a^{b}=u^{a} \nabla_{a} u^{b}$ is the fluid acceleration. Here we have assumed that the fluid velocity $u^{a}$ is aligned with a worldvolume Killing vector field $\mathbf{k}^{a}$ with modulus $\mathbf{k}$, that is, $u^{a}=\mathbf{k}^{a} / \mathbf{k}$ and furthermore that for any stationary configuration one must have that $\mathcal{L}_{\mathbf{k}} \mathbb{T}=0$ for an arbitrary tensor $\mathbb{T}$. Eq. (4.21) is solved by requiring the local temperature $\mathcal{T}$ and chemical potential $\Phi_{p}$ to satisfy

$$
T=\mathbf{k} \mathcal{T}, \quad \Phi_{\mathrm{H}}=\mathbf{k}\left(\Phi_{p}-\mathcal{C}_{1 a} u^{a}\right),
$$

where $T$ is the constant global temperature and $\Phi_{\mathrm{H}}$ the constant global chemical potential. When the background gauge field $\mathcal{C}_{1}$ vanishes, this reduces to the solution found in [18].

Using the relation between local temperature, horizon radius and chemical potential given in eqs. (C.3) and (C.5) in appendix C.1 together with (4.22) and (4.19), the partition function (4.7) takes the form

$$
\ln \mathcal{Z}=\beta \frac{\Omega_{(n+1)}}{16 \pi G}\left(\frac{n}{4 \pi T}\right)^{n} \int_{\mathcal{B}_{p}}{ }{ }_{(p)} \mathbf{k}^{n}\left(1-\frac{\Phi_{\mathrm{H}}^{2}}{N \mathbf{k}^{2}}-\frac{\left(\mathcal{C}_{1 a} u^{a}\right)^{2}}{N}\right)^{\frac{N n}{2}},
$$

where we have used (4.22) to replace terms containing $\Phi_{p}$. For consistency we note that, using (4.23), we can easily extract the electric current, i.e.,

$$
J_{1}^{a}=-\frac{\partial \ln \mathcal{Z}}{\partial \Phi_{p}} \frac{\partial \Phi_{p}}{\partial \mathcal{C}_{1 a}}=\mathcal{Q} u^{a},
$$

and also the correct perfect fluid stress-energy tensor

$$
T^{a b}=P \gamma^{a b}+\left(n P+\mathcal{Q} \Phi_{p}\right) u^{a} u^{b}=P \gamma^{a b}+(\epsilon+P) u^{a} u^{b},
$$

in agreement with appendix C.2. The equations of motion that follow from varying (4.23) by an arbitrary diffeomorphism are exactly those of (4.11) and (4.12) for $q=0$.

Finally, we consider changing to another ensemble that resembles the usual coupling of charged point particles moving in an external electric field where the total electric charge is held constant. This can be done by performing a global Legendre transformation by adding a term of the form $Q_{1} \Phi_{\mathrm{H}}$ to (4.23) with $Q_{1}$ being the total electric charge given by

$$
Q_{1}=\int_{\mathcal{B}_{p}} \mathcal{Q} u^{a} n_{a}, \quad n_{a}=\frac{\xi_{a}}{R_{0}},
$$

where $\xi^{a} \partial_{a}$ is the worldvolume Killing vector associated with time translations and $R_{0}$ its norm. The partition function (4.23) becomes

$$
\ln \mathcal{Z}=\beta\left(-\int_{\mathcal{B}_{p}}{ }^{\star}{ }_{(p)} P+Q_{1} \Phi_{\mathrm{H}}\right)=\beta\left(\int_{\mathcal{B}_{p}{ }^{\star}(p)}(\epsilon-\mathcal{T} s)+\int_{\mathcal{B}_{p}}{ }^{\star}(p) \mathcal{Q} u^{a} \mathcal{C}_{1 a}\right),
$$

where the energy density $\epsilon$, temperature $\mathcal{T}$ and entropy density $s$ are given in eqs. (C.7) and (C.3). The partition function (4.27) yields the same equations of motion as (4.23) as long as variations are taken at constant global electric charge $Q_{1}$. 


\subsection{External couplings for black branes carrying $q=p$-brane charge}

In this section we focus on the more complicated case of black brane solutions (3.1) to the action (2.4) with external sources of gauge $\mathcal{C}_{q+1}$ and dilaton field $\varphi$. In order to evaluate the Euclidean on-shell action we consider the general result (4.16) for $q=p$. In such case the Euclidean action over a $D$-dimensional region $Y$ with boundary $\partial Y$ becomes

$$
I_{\mathrm{E}}=\frac{1}{8 \pi G} \int_{\partial Y} \star[K]-\frac{1}{16 \pi G} \frac{(p+1)}{(D-2)} \int_{\partial Y} e^{a_{p} \phi} C_{p+1} \wedge \star F_{p+2},
$$

where the boundary $\partial Y$ is chosen to be a constant radial slice at infinity in the geometry of (3.1). Since this case is qualitatively different than the previous example, we first consider the situation for which no background gauge or dilaton fields are present, i.e., $\mathcal{C}_{q+1}=\varphi=0$. In order to evaluate (4.17) we consider the gauge field and its field strength near infinity using (3.1),

$$
\begin{aligned}
C_{p+1} & =\left(-\frac{16 \pi G Q_{p}}{n r^{n}}-\Phi_{p}\right) \star_{(p+1)} 1+\mathcal{O}\left(\frac{r_{0}}{r}\right)^{2 n}, \\
F_{p+2} & =16 \pi G \frac{Q_{p}}{r^{(n+1)}} d r \wedge \star_{(p+1)} 1+\mathcal{O}\left(\frac{r_{0}}{r}\right)^{2 n+1},
\end{aligned}
$$

where we have shifted the gauge field, via a gauge transformation, by subtracting the horizon chemical potential $\Phi_{p}=\sqrt{N} \tanh \alpha$ so that the gauge field is regular at the horizon [46] as in the example of the previous section.

Direct evaluation of (4.17) and using (4.29) leads to the same form of the Euclidean on-shell action (4.19) as in the previous example. In this case, the integrand of (4.19) is identified with the local Gibbs free energy $\mathcal{G}$ of the brane and not with the pressure (see eq. (C.2)). In order to re-express it in terms of the background sources as in (4.7), we demand gauge invariance and worldvolume diffeomorphism invariance. The former implies that the electric current $J_{p+1}$ as given in (C.1) is conserved which in turn implies that the total dipole charge $Q_{p}$ is constant on the worldvolume, i.e.,

$$
\partial_{a} Q_{p}=0
$$

This condition suggests that the thermodynamic ensemble of the Euclidean action (4.19) at fixed global temperature and global chemical potential is not the appropriate one in order to directly deal with the worldvolume conservation equations. For this reason, one changes to a new ensemble where the total charge $Q_{p}$ is held constant instead of the global chemical potential. As in [20], we perform a local Legendre transformation by adding $\Phi_{p} Q_{p}$ to the Gibbs free energy $\mathcal{G}$. By doing so, the Euclidean action (4.19) at fixed $Q_{p}$, which we refer to as $\tilde{I}_{\mathrm{E}}$, is given by

$$
\tilde{I}_{\mathrm{E}}=-i \beta \int_{\mathcal{B}_{p}} \star_{(p)}\left(\frac{\Omega_{(n+1)}}{16 \pi G} r_{0}^{n}+\Phi_{p} Q_{p}\right)=-i \beta \int_{\mathcal{B}_{p}}{ }{ }_{(p)} P
$$


where the pressure $P$ is given in eq. (C.2) of appendix C.1. However, since (4.31) must hold globally, we readily identify the global chemical potential $\Phi_{\mathrm{H}}$ as $^{14}$

$$
\Phi_{\mathrm{H}}=\int_{\mathcal{B}_{p}}{ }^{*}(p) \Phi_{p} .
$$

We now turn to the requirement of worldvolume diffeomorphism invariance, which in the absence of external backgrounds fields is given by (4.11), i.e., $\nabla_{a} T^{a b}=0$. Assuming the conservation of the entropy current $J_{s}^{a}=s u^{a}$, this set of equations is solved by

$$
T=\mathbf{k} \mathcal{T}, \quad u^{a}=\frac{\mathbf{k}^{a}}{\mathbf{k}} .
$$

We note that $\Phi_{p}$ is not a local degree of freedom of the fluid because it is completely determined by the condition (4.30) and therefore does not contribute to the solution (4.33). Given the solution (4.33) and using the relation between the local temperature $\mathcal{T}$ of the black brane (3.1) in terms of the horizon radius $r_{0}$ and chemical potential $\Phi_{p}$ (eqs. (C.3) and (C.5) in appendix C.1) the partition function (4.7) takes the form

$$
\ln \mathcal{Z}=\beta \frac{\Omega_{(n+1)}}{16 \pi G}\left(\frac{n}{4 \pi T}\right)^{n} \int_{\mathcal{B}_{p}}{ }_{(p)} \mathbf{k}^{n}\left(1-\frac{\Phi_{p}^{2}}{N}\right)^{\frac{N n}{2}}\left(1+\frac{n \Phi_{p}^{2}}{1-\frac{\Phi_{p}^{2}}{N}}\right),
$$

and should be extremized at fixed $T$ and $Q_{p} \cdot{ }^{15}$ Alternatively, since we have identified the global chemical potential (4.32) one may perform the inverse Legendre transformation of (4.31) in order to obtain a variational principle at fixed $T$ and $\Phi_{\mathrm{H}}$ as in [20].

Adding external background fields. We now consider introducing background values for the dilaton and gauge fields. As explained in section 3.1, a constant shift in the dilaton field is a symmetry of the action (2.4) and leads to a rescaling of the gauge field, i.e.,

$$
\phi \rightarrow \phi+\varphi, \quad C_{p+1} \rightarrow e^{-\frac{a_{p}}{2} \varphi} C_{p+1}
$$

In turn this implies that the chemical potential and the electric charge are rescaled according to

$$
\boldsymbol{\Phi}_{p}=e^{-\frac{a_{p}}{2} \varphi} \Phi_{p}, \quad \boldsymbol{Q}_{p}=e^{\frac{a_{p}}{2} \varphi} Q_{p}
$$

such that the product $\boldsymbol{\Phi}_{p} \boldsymbol{Q}_{p}=\Phi_{p} Q_{p}$ remains invariant. We then add a constant gauge field $\mathcal{C}_{q+1}$ such that $C_{p+1} \rightarrow C_{p+1}+\mathcal{C}_{q+1}$. Analogously to the previous case, constant shifts of the gauge field do not affect the evaluation of the Euclidean on-shell action while constant shifts of the dilaton only modify the result via (4.36). This is expected since both these shifts are symmetries of the action (2.4). Therefore, once again, the Euclidean on-shell action is given by (4.19).

\footnotetext{
${ }^{14}$ Since in order to obtain the global chemical potential one must integrate over the local one, $\Phi_{p}$ is better understood as a density of chemical potential on the brane, analogously to the energy density $\epsilon$ or the entropy density $s$.

${ }^{15}$ It may not be evident from (4.34) but the correct perfect fluid stress-energy tensor (C.1) follows from (4.34) using (4.10) and noting that $\left.\left(\delta \ln \mathcal{Z} / \delta \Phi_{p}\right)\right|_{T, Q_{p}}=0$.
} 
The presence of background fields, however, changes significantly the analysis. Demanding gauge invariance now implies that the modified charge is conserved along the worldvolume, i.e., $\partial_{a} \boldsymbol{Q}_{p}=0$ since the electric current is now given by (3.13). As in the case where no external background fields were present, one should now change to a new ensemble where $\boldsymbol{Q}_{p}$ is held constant instead of the global chemical potential but prior to do so we will consider the requirement of diffeomorphism invariance (4.11) which in this case reads

$$
\nabla_{a} T^{a b}=j_{\phi} \partial^{b} \varphi
$$

where $j_{\phi}$ is given in (3.13). We note that there is no Lorentz force because $\mathcal{C}_{p+1}$ is a top-form from the worldvolume point of view. As we will see below, this implies that the background field $\mathcal{C}_{p+1}$ will not affect the requirements due to worldvolume diffeomorphism invariance but it will contribute to changes in the global chemical potential. We now proceed and try to find a stationary solution to (4.37) for arbitrary background sources. Projecting (4.37) along $u_{b}$ leads to a vanishing l.h.s. assuming the conservation of the entropy current and therefore we obtain the condition $u^{a} \partial_{a} \varphi=0$. This condition suggests that one must, as in the previous cases, choose $u^{a}=\mathbf{k}^{a} / \mathbf{k}$ and in fact we demonstrate in appendix D that this must indeed be the case in order to have a fluid configuration that does not dissipate. On the other hand, the projection of (4.37) perpendicular to the fluid flows leads to

$$
\mathcal{T} s P_{b}^{c}\left(\partial^{b} \ln \mathcal{T}+a^{b}\right)=j_{\phi} P^{c}{ }_{b}^{b} \varphi
$$

We see that the driving force due to a spatially varying dilaton must be compensated by a modification of the local temperature $\mathcal{T}$ compared to (4.33) since the dilaton current $j_{\phi}$ is non-vanishing at leading order. Note that this case is qualitatively different than the case considered in [10] since there $j_{\phi}$ appears at first order in derivatives and hence plays no role at leading order. In order to solve (4.38) we denote the $m$ worldvolume directions perpendicular to $u_{a}$ collectively by $\tilde{\sigma}=\left\{\tilde{\sigma}_{1}, \ldots, \tilde{\sigma}_{m}\right\}$ and make the ansatz

$$
\mathcal{T}=\frac{T}{\mathbf{k}} f(\tilde{\sigma})
$$

for constant global temperature $T$ and for some function $f(\tilde{\sigma})$ to be determined. Introducing this ansatz into (4.38) leads to ${ }^{16}$

$$
\mathcal{T} s \partial_{c} \ln f(\tilde{\sigma})=j_{\phi} \partial_{c} \varphi(\tilde{\sigma}) .
$$

It is now imperative to note that the product $\mathcal{T} s$, as well as the dilaton current $j_{\phi}$, is a function of the temperature $\mathcal{T}$ and the global charge $\boldsymbol{Q}_{p}$ for the specific case that we are considering. Obtaining the dependence of these quantities in terms of $\mathcal{T}$ is not straightforward as it demands obtaining $\boldsymbol{\Phi}_{p}$ in terms of $\boldsymbol{Q}_{p}$. This can be done analytically in appropriate small or large charge limits as in $[28,29]$ and for specific values of $n$. Nevertheless, the final result is always dependent on $\mathcal{T}$ and therefore from (4.39) and (4.40) the function $f(\tilde{\sigma})$ will always depend non-trivially on the global temperature $T$. When

\footnotetext{
${ }^{16}$ Note that $\varphi$ can also depend on the transverse scalars $X^{i}$ without affecting the analysis that we are carrying out.
} 
such dependence is introduced in the partition function (4.7) then direct evaluation of the entropy using (4.10) would lead to modifications to the entropy current $J_{s}^{a}=s u^{a}$ which are not present at leading order. ${ }^{17}$ This forces us to conclude that there are no stationary solutions to (4.37) with current $j_{\phi}$ as given in (3.13) and with a spatially varying dilaton $\varphi(\tilde{\sigma})$ along the worldvolume directions. In turn, we conclude that there are no regular stationary black holes constructed from fluid-type deformations of (3.1) with a spatial varying dilaton background field along worldvolume directions, though it can depend non-trivially on the transverse scalars $X^{i}$. Therefore, the solution (4.33) still holds with the further requirement that $\partial_{a} \varphi=0$ when the temperature $\mathcal{T}$ is non-zero. At extremality $(\mathcal{T}=0)$ the result is different. In this case, the stress-energy tensor becomes $T^{a b}=-e^{-a_{p} \varphi / 2} \boldsymbol{Q}_{p} \gamma^{a b}$ and therefore (4.37) is automatically satisfied.

Given the stationary solution at finite temperature just obtained, we have all the necessary ingredients to write the partition function in terms of the background sources. However, as mentioned earlier, the presence of a background top-form gauge field does not affect (4.33) but can contribute to changes in the global chemical potential (4.32). We parametrize this ignorance by considering an additional contribution $\tilde{\boldsymbol{\Phi}}_{p}$ to the global chemical potential (4.32) such that

$$
\Phi_{\mathrm{H}}=\int_{\mathcal{B}_{p}} \star_{(p)} \boldsymbol{\Phi}_{p}+\int_{\mathcal{B}_{p}} \tilde{\boldsymbol{\Phi}}_{p} .
$$

We now consider performing a global Legendre transformation in the Euclidean action (4.19) by adding a term of the form $\boldsymbol{Q}_{p} \Phi_{\mathrm{H}}$. The partition function becomes

$$
\ln \mathcal{Z}=\beta\left(\int_{\mathcal{B}_{p}}{ }^{\star}(p) \mathcal{G}+\Phi_{\mathrm{H}} \boldsymbol{Q}_{p}\right)=-\beta \int_{\mathcal{B}_{p}}\left({ }^{\star}(p) P-\boldsymbol{Q}_{p} \tilde{\boldsymbol{\Phi}}_{p}\right) .
$$

Since the background gauge field $\mathcal{C}_{p+1}$ did not affect (4.33), the pressure $P$ has no dependence on $\mathcal{C}_{p+1}$. Therefore, demanding consistency with (4.10) we must require that

$$
J_{p+1}^{a_{1} \ldots a_{p+1}}=-\frac{1}{\beta} \frac{\delta \ln \mathcal{Z}}{\delta \mathcal{C}_{p+1 a_{1} \ldots a_{p+1}}}=-\boldsymbol{Q}_{p} \frac{\delta \tilde{\boldsymbol{\Phi}}_{p}}{\delta \mathcal{C}_{p+1 a_{1} \ldots a_{p+1}}}=\boldsymbol{Q}_{p} \epsilon^{a_{1} \ldots a_{p+1}},
$$

where $\epsilon^{a_{1} \ldots a_{p+1}}$ is the Levi-Civita tensor on the $(p+1)$-dimensional worldvolume. Eq. (4.43) has a unique straightforward solution, namely, $\tilde{\boldsymbol{\Phi}}_{p}=-\mathbb{P}\left[\mathcal{C}_{p+1}\right]$ where $\mathbb{P}\left[\mathcal{C}_{p+1}\right]$ is the pullback of the gauge field onto the worldvolume. Finally, using the relations between local temperature and chemical potential in appendix C.1, the solution (4.33), $\partial_{a} \varphi=0$ and $\tilde{\boldsymbol{\Phi}}_{p}=-\mathbb{P}\left[\mathcal{C}_{p+1}\right]$ we obtain the partition function in terms of the background sources

$$
\ln \mathcal{Z}=-\beta \int_{\mathcal{B}_{p}}\left(\star_{(p)} P+\boldsymbol{Q}_{p} \mathbb{P}\left[\mathcal{C}_{q+1}\right]\right)
$$

where the pressure $P$ is given by

$$
P=-\frac{\Omega_{(n+1)}}{16 \pi G}\left(\frac{n}{4 \pi T}\right)^{n} \mathbf{k}^{n}\left(1-\frac{e^{a_{p} \varphi} \boldsymbol{\Phi}_{p}^{2}}{N}\right)^{\frac{N n}{2}}\left(1+\frac{n e^{a_{p} \varphi} \boldsymbol{\Phi}_{p}^{2}}{1-\frac{e^{a_{p} \varphi} \mathbf{\Phi}_{p}^{2}}{N}}\right) .
$$

\footnotetext{
${ }^{17}$ Besides this general argument, we have not been able to find solutions to (4.40) even in simple cases of constant driving forces.
} 
When no background dilaton fields are present, the partition function (4.44) was the one used in $[28,29]$ to study giant graviton configurations at finite temperature. As a consistency check, consider obtaining the dilaton current from (4.44) using (4.10), one finds ${ }^{18}$

$$
j_{\phi}=-\left.\frac{\delta \ln \mathcal{Z}}{\delta \varphi}\right|_{T, \boldsymbol{Q}_{p}}=\frac{a_{p}}{2} Q_{p} \Phi_{p},
$$

in agreement with (3.13).

The extremal limit. The partition function (4.44) must reduce to the DBI action in the presence of a background dilaton and gauge field once the extremal limit $\mathcal{T} \rightarrow 0$ is taken. This limit requires that $r_{0} \rightarrow 0, \alpha \rightarrow \infty$ and $T \rightarrow 0$ while keeping the total charge $\boldsymbol{Q}_{p}$ fixed. Therefore, at extremality, we find the following limiting behavior for the fluid pressure $P \rightarrow-e^{-a_{p} \varphi / 2} \boldsymbol{Q}_{p}$. Identifying $\boldsymbol{Q}_{p}$ with the brane tension $T_{p}$ such that $\boldsymbol{Q}_{p}=T_{p}$ we obtain the DBI action in the form

$$
S=-\frac{1}{\beta} \int d t \ln \mathcal{Z}=-T_{p} \int_{\mathcal{W}_{p+1}} d^{p+1} \sigma e^{-a_{p} \varphi / 2} \sqrt{-\gamma}+T_{p} \int_{\mathcal{W}_{p+1}} \mathbb{P}\left[\mathcal{C}_{q+1}\right],
$$

as expected. We note that this action is valid for arbitrary background dilaton field $\varphi\left(\sigma, X^{i}\right)$ contrary to the finite temperature case where $\varphi$ cannot have any dependence on the coordinates $\sigma$ in stationary equilibrium.

\section{Conclusions}

The ultimate objective of this work is a systematic construction of black hole solutions in appropriate long-wavelength expansion schemes in arbitrary (super)gravity theories. In the present paper we continued work in this direction in the context of the blackfold formalism to include generic effects of the asymptotic background that arise from curvature, and/or fluxes of general matter fields. Focusing on the constraint equations of the gravitational system at first order in the long-wavelength expansion we derived an effective hydrodynamic description that involves fluids propagating under the influence of external forces. The resulting equations are the dynamical equations of forced blackfolds. These equations describe a part of the full dynamics of the putative complete (super)gravity solution. We conclude with a few remarks on the (super)gravity problem, the regimes of the sought-after perturbative expansions, and some of the key issues that arise in the presence of generic asymptotic backgrounds (that are less elaborated upon in the existing literature).

\subsection{Comments on the long-wavelength expansions of the blackfold approach}

Elements and formulation of the (super)gravity problem. The specific problem that we want to consider in (super)gravity starts with the following ingredients:

(a) We are given an arbitrary gravitational action in $D$ spacetime dimensions $(D>4)$. Besides the metric, this action may involve a variety of other fields, e.g. matter fields and abelian gauge fields that are common in supergravities.

\footnotetext{
${ }^{18}$ Note that by consistency we also find $\Phi_{\mathrm{H}}=-\left.\left(\partial \ln \mathcal{Z} / \partial \boldsymbol{Q}_{p}\right)\right|_{T}$ where $\Phi_{\mathrm{H}}$ is given in (4.41) with $\tilde{\boldsymbol{\Phi}}_{p}=$ $-\mathbb{P}\left[\mathcal{C}_{p+1}\right]$.
} 
(b) An exact (black) p-brane solution of the equations of motion of this action with specified, but arbitrary asymptotics, is known. We assume that the $p$-brane solution has Killing isometries along $m \leq p+1$ non-compact worldvolume directions, and is characterized by $\ell$ independent free parameters, e.g. thermodynamic parameters like the mass, or other charges. The $m$ symmetries of the solution are a subset of the symmetries of the asymptotic background. To avoid potential backreaction issues to the asymptotic background we also assume that the codimension of the $p$-brane solution is appropriately high, typically greater than two, $D-p-1>2$.

Our goal is to construct a larger class of inhomogeneous $p$-brane solutions with the same asymptotics, where the $m$ symmetries of the solution in $(b)$ are broken. The new solutions are continuous deformations of the (partially) homogeneous solution in point $(b)$.

Long-wavelength deformations. The $m$ non-compact symmetric directions imply the potential existence of symmetry-breaking deformations in a long-wavelength regime. A natural subclass of such deformations can be attacked with semi-analytic methods that promote the $\ell$ free parameters of the leading order exact solution $(b)$ to arbitrary slowlyvarying functions of the $m$ spacetime coordinates along which we seek to break the symmetry. With an appropriate ansatz for the (super)gravity fields based on an order-by-order deformation of the leading order solution one aims to construct less symmetric solutions perturbatively in a scheme of small derivative expansions. The best studied and most successful application of this logic in gravity has focused on large AdS black holes, where it leads to the fluid/gravity correspondence. Applications in a wider setting constitute the basis of the blackfold formalism.

Clearly, the extent of the gravitational dynamics that can be captured in the above analysis depends on the form of the leading order solution and the ansatz that is employed to study deformations around it.

The ansätze that were described in the main text and are usually employed in the context of the blackfold formalism may not cover in general the full set of available longwavelength dynamics. In [30] it was emphasized that the blackfold approach captures the effective long-wavelength dynamics of abelian singleton degrees of freedom, and may contain only partial information about long-wavelength dynamics of the microscopic nonabelian degrees of freedom. From a gravitational point of view, a better understanding of the degrees of freedom that dominate the long-wavelength dynamics can be obtained by studying the spectrum of quasinormal modes of the black brane solution. A complete analysis of quasinormal modes of black branes in general spacetimes is currently missing.

'Exact brane' applications of the blackfold approach. The (super)gravity problem that was formulated above provides from the start two exact gravitational solutions: a solution that fixes the asymptotic spacetime (background solution), and a $p$-brane solution with a suitable codimension (leading order solution of the subsequent expansion scheme). The leading order solution asymptotes to the background solution at large distances along a radial direction. In general, the two solutions have different symmetries and are characterized by different characteristic scales. Let us call $L$ the characteristic scale of the 
background solution, and $r_{H}$ the characteristic scale of the leading order solution. $r_{H}$ is a characteristic horizon scale, e.g. it can be the charge radius of a charged $p$-brane (the AdS radius for a solution with an AdS near-horizon region), or the Schwarzschild radius for a black $p$-brane at finite temperature. ${ }^{19}$ Carrying out the long-wavelength deformation analysis exactly in the ratio $r_{H} / L$ will be dubbed the 'exact brane' application of blackfolds.

In this exercise the deformation of the leading order solution that we seek introduces a third scale into the problem: the scale $\mathcal{R}$ of deformations. Since we are interested in long-wavelength deformations, by definition we require the hierarchy of scales

$$
r_{H} \ll \mathcal{R}
$$

Notice that the background scale $L$ does not enter in this inequality, because the leading order solution is exactly known for all values of $r_{H}$ and $L$.

The perturbative construction of long-wavelength deformations of the leading order solution can be pursued with the use of a suitable scheme of matched asymptotic expansions (MAEs) (see refs. [11-13, 22] for concrete applications of this method in the context of blackfold constructions). In a MAE the (super)gravity equations are solved separately in a near-zone region $(r \ll \mathcal{R})$, and a far-zone region $\left(r \gg r_{H}\right)$. The large hierarchy of scales (5.1) guarantees the existence of a large intermediate overlap region $\left(r_{H} \ll r \ll \mathcal{R}\right)$ where the integration constants of the near- and far-zone solutions are matched.

In this context, part of the gravitational equations (constraint equations) result naturally to a $(p+1)$-dimensional effective hydrodynamic description of the collective mode dynamics of the resulting $p$-brane solution. This description, which was the main theme in this paper, is formulated as a set of conservation equations for appropriate currents.

These currents are evaluated in the overlap zone, deep in the asymptotic region where one can position the screen of the effective description. In general, they depend non-trivially on the details of the background solution and its scale $L$. As one proceeds order-by-order in the derivative expansion these currents receive higher-derivative corrections, but the conservation equations are always formulated as equations of a $(p+1)$-dimensional fluid on a dynamical (elastic) hypersurface propagating in the fixed asymptotic supergravity background that does not get any corrections in the expansion.

'Exact brane' applications and open/closed string duality. Ref. [30] recently argued that this effective description of collective mode dynamics in gravity is related holographically to the effective description of a dual non-gravitational higher-spin theory (open string field theory in the case of D-branes) via a general open/closed string duality. For extremal $p$-brane solutions in flat space, it was further anticipated that the derivative corrections of the effective hydrodynamic equations in gravity are dual at all orders to the higher-derivative corrections of the abelian Dirac-Born-Infeld (DBI) action, which can be computed independently in classical open string theory.

\footnotetext{
${ }^{19}$ The generic situation may involve further characteristic scales with a more complicated pattern of regimes. We will shortly address such an example below. For the moment we keep a minimum number of scales to exhibit clearly the basics of the construction.
} 
Three interesting practical aspects of this connection are the following. First, it is interesting that gravitational solutions in asymptotically flat space are carrying information about the higher-spin degrees of freedom of the dual open string field theory. Second, exact solutions in gravity or the dual open string theory are providing non-perturbative completions of the hydrodynamic blackfold derivative expansion. Third, when the exercise is performed in curved/fluxed asymptotic backgrounds in the 'exact brane' application (i.e. exactly in $\left.r_{H} / L\right)$, the effective long-wavelength description of the collective mode dynamics is expected to provide an interesting deformation of DBI-like actions that incorporates information about open-closed string couplings beyond the standard ones in weak coupled open string theory. Finite temperature corrections are producing further deformations of DBI-like actions.

Multiple expansions and further approximations in the blackfold approach. Frequently, in practical applications one has to deal with complicated gravitational configurations where some of the exact solution prerequisites of the above (super)gravity problem are not known. For example, the exact leading order solution is not a priori known, and has to be constructed from scratch. In that case we cannot proceed with the 'exact brane' application of the blackfold formalism that was outlined above. Instead, one can attempt to employ a secondary parallel expansion scheme that reconstructs the leading order solution perturbatively around a solution in a different asymptotic background [7]. As an illustration, let us consider two examples emphasizing the interplay of different scales and the multiple expansions associated with them.

As a first concrete example consider the construction of a black string solution in AdS. In this case the background characteristic scale is the radius $L$ of the asymptotic AdS solution. Since an exact leading order black string solution in AdS is not known it was pointed out in [14] that one could proceed perturbatively in the limit

$$
r_{H} \ll \min (\mathcal{R}, L)
$$

Besides the derivative expansion in the limit $r_{H} / \mathcal{R} \ll 1$, (5.1), which is characteristic of the 'exact brane' application of the blackfold formalism, the inequality (5.2) allows us to implement a second parallel expansion in the small ratio $r_{H} / L$. At first order in this second expansion the leading order solution of the blackfold expansion can be approximated locally (in the transverse space) by the well-known uniform black string solution in flat space. From the point of view of the leading order blackfold equations, in this regime one describes how a black string probe propagates on the AdS background. For details of this approximation we refer the reader to refs. [14, 19]. More generally, the cases that we considered in section 3 are of this type, since the charged black brane solutions we use as input are asymptotically flat.

As another example consider the case of a double-centered D3-brane solution in tendimensional type IIB supergravity. Viewing one of the centers as the background spacetime we can ask whether it is possible to add perturbatively the second center to obtain general solutions describing how two stacks of D3-branes interact in supergravity. We note immediately that this is a case where the single-centered D3-brane solution cannot be viewed as 
a proper asymptotic background according to the definition of the problem posed in the beginning. It is clear that the second center backreacts to deform the first center.

In the 'exact brane' application of the blackfold formalism in this example the true background solution is the asymptotic flat space, and one would have to begin with an exact double-centered leading order solution. For example, extremal solutions could be constructed perturbatively around the planar double-centered supersymmetric solution with harmonic function

$$
H=1+\frac{L^{4}}{|\vec{x}-\vec{\Delta}|^{4}}+\frac{r_{H}^{4}}{|\vec{x}|^{4}} .
$$

In this expression, we call $L$ the near-horizon AdS radius of the first center at $\vec{x}=\vec{\Delta}$, and $r_{H}$ the near-horizon AdS radius of the second center at $|\vec{x}|=0 . \vec{x}$ is a 6-vector parameterizing the six-dimensional space transverse to the planar D3-brane worldvolumes of the leading order solution. The blackfold derivative expansion would proceed by promoting the scales $L, r_{H}$, and the vector of relative positions $\vec{\Delta}$ to slowly varying functions of the 3 brane worldvolume coordinates. For this expansion we would simply require the hierarchy of scales

$$
L, r_{H},|\vec{\Delta}| \ll \mathcal{R}
$$

It is clear from (5.3) that in regions where $|\vec{x}| \ll|\vec{\Delta}|$ (the vicinity of the second center) we can approximate the leading order solution in terms of a single-centered uniform 3-brane in flat-space. In the region $r_{H} \ll|\vec{x}| \ll|\vec{\Delta}|$ the space asymptotes to the background of the first center. Then, in the spirit of (5.2) we could employ a multiple expansion in the limit

$$
\begin{gathered}
r_{H} \ll|\vec{\Delta}|, \\
r_{H} \ll \min (\mathcal{R}, L) .
\end{gathered}
$$

At first order in the power-series expansion in terms of the small ratios $r_{H} /|\vec{\Delta}|$ and $r_{H} / L$ we can phrase the blackfold expansion (namely the expansion in powers of $r_{H} / \mathcal{R}$ ) in terms of a deformed flat-space D3-brane (representing the second center) that propagates in the background of the first center. However, unlike the case of the inequality (5.2), at higher orders of $r_{H} /|\vec{\Delta}|$ and $r_{H} / L$ the backreaction of the second-center D3-blackfold to the first center background has to be included.

Multiple expansions versus open + closed string theory. As we mentioned previously, in the case of an exactly known leading order solution and the single expansion in (5.1), the collective mode (blackfold) equations are phrased as a lower-dimensional theory on a screen propagating in a fixed background. This lower-dimensional theory has a conjectured open string dual [30]. The open and closed string pictures are complementary equivalent descriptions of the same dynamics.

In contrast, the above-mentioned multiple expansions, e.g. when (5.2) holds, have a closer resemblance to an interacting system of both open and closed strings. In the associated derivative expansion schemes in gravity, the effective theory on the lower-dimensional screen interacts order-by-order with a dynamical gravitational theory in the bulk. The dynamics of the 'open/closed string' couplings in the gravity-induced effective description 
are expressing the parallel expansion in $\mathcal{R} / L$ and the associated backreaction effects of the probe to the bulk. It would be very interesting to understand better how such multiple expansions proceed in gravity, and how the bulk-boundary interactions are encoded in the effective long-wavelength description. From a purely effective field theory point of view, when the backreaction effects are included one has to deal with related self-gravity effects. Typically, such effects lead to divergences. Since in the context of the expansions of the full gravitational equations one has a concrete underlying system of equations that characterize well-defined gravity solutions, it is natural to expect that a proper understanding of the gravity-induced effective description knows how to deal properly with such divergences. It is interesting to examine this aspect in detail. We emphasize again, that no backreaction effects are expected in the 'exact brane' application of the blackfold formalism where the asymptotic gravity solution is fixed and non-dynamical at all orders in the long-wavelength derivative expansion.

\subsection{Open problems}

Let us conclude with the summary of a few interesting open problems that constitute a natural continuation of the work presented in this paper.

Effective actions for arbitrary background field configurations. In section 4 we have derived, using diffeomorphism and gauge invariance, the general form of the constraint equations of systems coupled to a background gauge field $\mathcal{C}_{q+1}$ and a dilaton $\varphi$. This, however, does not exhaust in any way the possible types of couplings and force terms that have been derived in section 2. In certain cases, it is straightforward to extend the analysis of section 4 to include further couplings. For example, in the case of type IIA/B supergravity, one may consider background solutions with $H_{3} \neq 0$ and probes without magnetic currents. In these cases, the hydrostatic partition function is easily generalized by adding further gauge fields of different ranks. However, once magnetic currents are turned on, it is necessary to consider couplings to the several Dirac branes involved as in [45]. Furthermore, if $H_{3}$ is non-vanishing, further work will be necessary in order to obtain force terms of the schematic form H.C.J. It would be of great interest to understand such examples in detail since they would allow us to study, for example, the DBI action and the PST action [34] at finite temperature in an arbitrary background in the spirit of $[17,23,28,29,32,33,55-59]$.

Effective actions at extremality and new supergravity solutions. The construction of new extremal supergravity solutions is particularly opportune technically, especially if some amount of supersymmetry is present. It was pointed out in [35] that a perturbative construction of supersymmetric solutions in a long-wavelength regime may lead to an interesting framework of $G$-structure deformations where the Killing spinor version of the constraint equations is related to $\kappa$-symmetry conditions.

It was further pointed out in [30], as we emphasized above, that the long-wavelength treatment of extremal (but not necessarily supersymmetric) $p$-brane solutions leads naturally to the DBI equations, well-known from open string theory. It would be interesting to extend this connection beyond the examples of [30] to include general open-closed string 
couplings of the DBI. It would also be interesting to explore what kind of deformations of the DBI action are induced in the supergravity-derived blackfold effective action in 'exact brane' applications of blackfolds where one goes beyond the usual probe equations derived from the use of approximations based on flat space $p$-brane solutions.

Further development of this formalism in supergravity could be useful in many applications that require the construction of complicated extremal supergravity solutions. A particular problem of interest, is the construction of new solutions describing the gravitational backreaction of massive configurations of D/M-branes. Recent applications to brane intersections in string/M-theory in flat space include [17, 23, 28, 29, 33, 55-57]. Solutions in supergravity backgrounds with fluxes have not been studied in this manner and it would be interesting to do so. For example, it would be interesting to examine the backreaction of anti-brane configurations in backgrounds with fluxes along the lines of recent work in this direction (see, for instance [60-62]). For example, one can ask about extremal D3-D5 blackfold constructions in the Klebanov-Strassler background [63] (in analogy to [60, 64, 65]), or anti-M2 blackfolds at the tip of Stenzel space in M-theory (in analogy to [66-68]). In all these cases, the real problem, which is also the central issue in the recent literature, has to do with the construction of the leading order solution (in the language of the previous subsection). The combination of recent results in the literature of anti-brane backreaction with MAE techniques frequently used in the blackfold formalism might be fruitful.

More examples of 'exact brane' applications; similarities with the tachyon-DBI derivation. In the formulation of the general supergravity problem in the beginning of subsection 5.1 we purposely included the case of leading order $p$-brane solutions with $m<p+1$ symmetric worldvolume directions. In such cases the leading order solution is already inhomogeneous in $p+1-m$ directions. For example, it could be time-dependent in a time-independent background. It would be interesting to find and examine an explicit example of this type. At this point one cannot help but notice the analogy of such a case with $S$-brane and rolling tachyon solutions in string theory $[69,70]$ and the corresponding derivation of the tachyon-DBI action as an open string long-wavelength effective field theory around the rapidly changing rolling tachyon solution [71].

Forcing and time-dependence. In the presence of a curved/fluxed background the typical solutions will be non-stationary solutions with a small number of symmetries, with the non-stationarity being driven by the external forcing. These effects will be manifested in the exact leading order solution and/or in the forced blackfold equations in multiple expansions. It is interesting to understand further the physics of such effects, and their implications in the construction of perturbative time-evolving black brane solutions in explicit cases; for example, in cases of black brane solutions moving in the vicinity of other black hole solutions.

Higher order corrections to effective actions. The type of effective actions considered in this paper were derived at leading order in a long-wavelength expansion. At higher orders, one must take into account derivative corrections due to fluid and elastic deformations. These corrections can be taken into account in a systematic way following $[54,72,73]$ 
and it would be extremely interesting to consider these in the case of multi-charged bound states in the presence of external backgrounds fields as well as in the presence of boundaries as in [74]. As advertised in [25], these corrections would account for the polarization properties of relativistic fluids encoded in the form of the electric and magnetic susceptibilities in the stress-energy tensor and electric/magnetic currents besides the Young modulus [21] and the piezoeletric moduli [24]. Of considerable interest would be to consider corrections due to possible quantum anomalies for theories with higher-form gauge fields.

We also note that to compute the response coefficients corresponding to such higher order corrections, one needs to have access to the full first-order corrected solution, i.e. solve all the field equations in the near-zone to first order (and not the subset of constraint equations considered in section 3 for particular examples). Performing this analysis (which was done for various types of blackfold constructions in e.g. [9, 11-15, 22]), even though challenging, would be interesting in its own right in order to fully show that to this order a solution exists that is regular on the horizon.

First law of thermodynamics in arbitrary background fields. The effective actions studied in this paper can be used to construct new stationary black hole solutions by solving the constraint equations in the presence of background fields for specific configurations. As exemplified in [75], if the resulting solutions are characterized by length scales associated with the background spacetime, these can be allowed to vary leading to new terms in the first law of thermodynamics involving integrated brane tensions - the dual thermodynamic quantities to the background length scales. However, here we have generalized these actions to also include background gauge and dilaton fields, which can in principle be characterized by several non-trivial length scales, e.g. as in the case of a non-trivial black hole solution playing the role of the background field configuration. These length scales can now be allowed to vary leading to new terms in the first law for which their dual thermodynamic quantities may be of interest to study.

\section{Acknowledgments}

We would like to thank Jyotirmoy Bhattacharya, Matthias Blau, Ori Ganor, Gianluca Grignani, Adolfo Guarino, Troels Harmark, Jelle Hartong, Gianluca Inverso, Cindy Keeler, Ricardo Monteiro and Marta Orselli for useful discussions. The work of VN was supported in part by European Unions Seventh Framework Programme under grant agreements FP7REGPOT-2012-2013-1 no 316165, and by European Unions Horizon 2020 Programme under grant agreement 669288-SM-GRAV-ERC-2014-ADG. The work of JA was supported by the ERC Starting Grant 335146 HoloBHC. JA acknowledges two short term mission grants to NBI, one by the Holograv network and another by the COST network. JG is supported by VILLUM FONDEN, research grant VKR023371. The work of NO is supported in part by the Danish National Research Foundation project "New horizons in particle and condensed matter physics from black holes". The work of AVP has been supported by The Danish Council for Independent Research - Natural Sciences (FNU), DFF-4002-00307. AVP gratefully acknowledge support from UC Berkeley where some of the research for this paper was carried out. 


\section{A Notation and conventions}

In this section we collect the notation and convention used throughout this paper. We define a generic $p$-form with components $A_{(p) \mu_{1} \ldots \mu_{p}}$ and its Hodge dual as

$$
\begin{aligned}
A_{(p)} & =\frac{1}{p !} A_{(p) \mu_{1} \ldots \mu_{p}} d x^{\mu_{1}} \wedge \ldots \wedge d x^{\mu_{p}} \\
\star A_{(p)} & =\frac{1}{p !(D-p) !} \epsilon_{\mu_{1} \ldots \mu_{D-p}}{ }^{\nu_{1} \ldots \nu_{p}} A_{(p) \nu_{1} \ldots \nu_{p}} d x^{\mu_{1}} \wedge \ldots \wedge d x^{\mu_{D-p}} .
\end{aligned}
$$

Furthermore, the wedge product of a $p$ - and $q$-form is defined as

$$
A_{(p)} \wedge B_{(q)}=\frac{1}{p ! q !} A_{(p)\left[\mu_{1} \ldots \mu_{p}\right.} B_{\left.(q) \nu_{1} \ldots \nu_{q}\right]} d x^{\mu_{1}} \wedge \ldots \wedge d x^{\mu_{p}} \wedge d x^{\nu_{1}} \wedge \ldots \wedge d x^{\nu_{q}}
$$

while the exterior derivative of a $p$-form is given by

$$
d A_{(p)}=\frac{(p+1)}{p !} \nabla_{\left[\mu_{1}\right.} A_{\left.(p) \mu_{2} \ldots \mu_{p+1}\right]} d x^{\mu_{1}} \wedge \ldots \wedge d x^{\mu_{p+1}} .
$$

We define the square of the $\mathrm{D}$-dimensional $\star$ operator acting on a $p$-form in space-times with Lorentzian signature as

$$
\star^{2}=(-1)^{p(D-p)+1} .
$$

We also introduce the star operator on the worldvolume $\star_{(p+1)}$ such that $\star_{(p+1)} 1=$ $\sqrt{-\gamma} d \sigma^{0} \wedge \ldots \wedge d \sigma^{p}$ as well as the star operator $\star_{(p)}$ on the spatial part of the worldvolume $\mathcal{B}_{p}$ such that ${ }_{(p)} 1=\sqrt{-\gamma} d \sigma^{1} \wedge \ldots \wedge d \sigma^{p}$. We also assume that the worldvolume topology is $\mathcal{W}_{p+1}=\mathbb{R} \times \mathcal{B}_{p}$ and hence that the determinant of the induced metric on the worldvolume can be decomposed as $\sqrt{-\gamma} d \sigma^{1} \wedge \ldots \wedge d \sigma^{p}=R_{0} d V_{(p)}$ where $R_{0}$ is the modulus of the Killing vector field $\xi^{a} \partial_{a}$ associated with worldvolume time translations.

\section{B Explicit form of type IIA/B probe brane equations}

In this appendix we restrict the equations of motion (2.18)-(2.19) and the probe brane equations (2.22)-(2.23) to the type IIA/B cases individually.

Type IIA supergravity. We consider type IIA supergravity by restricting the equations of motion (2.18)-(2.19) to $q=0,2,4,6$. When all currents vanish, these equations can be obtained from the action ${ }^{20}$

$$
\begin{aligned}
I= & \frac{1}{16 \pi G} \int_{\mathcal{M}_{10}}\left[\star R-\frac{1}{2} d \phi \wedge \star d \phi-\frac{1}{2} e^{-\phi} H_{3} \wedge \star H_{3}-\frac{1}{2} \sum_{q=0,2} e^{a_{q} \phi} \tilde{F}_{q+2} \wedge \star \tilde{F}_{q+2}\right] \\
& -\frac{1}{32 \pi G} \int_{\mathcal{M}_{10}} C_{3} \wedge H_{3} \wedge F_{4},
\end{aligned}
$$

\footnotetext{
${ }^{20}$ We are using the conventions of [44] but we used the equivalence of Chern-Simons terms $\int B_{2} \wedge F_{4} \wedge F_{4}=$ $\int C_{3} \wedge H_{3} \wedge F_{4}$ up to boundary terms.
} 
while in the presence of sources the equations of motion become ${ }^{21}$

$$
\begin{array}{r}
d\left(e^{-\phi} \star H_{3}-e^{\phi / 2} \star \tilde{F}_{4} \wedge C_{1}-\frac{1}{2} C_{3} \wedge F_{4}\right)=-16 \pi G \star \mathbf{j}_{2}, \\
d\left(e^{3 \phi / 2} \star F_{2}\right)+e^{\phi / 2} H_{3} \wedge \star \tilde{F}_{4}=16 \pi G \star \mathbf{J}_{1}, \\
d\left(e^{\phi / 2} \star \tilde{F}_{4}\right)+H_{3} \wedge \tilde{F}_{4}=16 \pi G \star \mathbf{J}_{3} .
\end{array}
$$

In turn, the equations of motion for the probe brane can be obtained by restricting (2.22) and hence we obtain

$$
\begin{aligned}
\nabla_{\mu} \mathbf{T}^{\mu \nu}= & \frac{1}{2 !} H_{3}^{\nu \mu_{1} \mu_{2}} \mathbf{j}_{2 \mu_{1} \mu_{2}}+\frac{e^{-\phi}}{6 !} H_{7}^{\nu \mu_{1} \ldots \mu_{6}} \mathbf{j}_{6 \mu_{1} \ldots \mu_{6}}+\mathbf{j}_{\phi} \partial^{\nu} \phi \\
& +F_{2}^{\nu \mu_{1}} \mathbf{J}_{1 \mu_{1}}+\left(\frac{1}{3 !} \tilde{F}_{4}^{\nu \mu_{1} \ldots \mu_{3}}-\frac{1}{2 !} H_{3}^{\nu \mu_{1} \mu_{2}} C_{1}^{\mu_{3}}\right) \mathbf{J}_{3 \mu_{1} \ldots \mu_{3}} \\
& +e^{\phi / 2}\left(\frac{1}{5 !} \tilde{F}_{6}^{\nu \mu_{1} \ldots \mu_{5}}-\frac{1}{2 \cdot 3 !} H_{3}^{\nu \mu_{1} \mu_{2}} C_{3}^{\mu_{3} \ldots \mu_{5}}\right) \mathcal{J}_{5 \mu_{1} \ldots \mu_{5}} \\
& +\frac{e^{3 \phi / 2}}{7 !} \tilde{F}_{8}^{\nu \mu_{1} \ldots \mu_{7}} \mathcal{J}_{7 \mu_{1} \ldots \mu_{7}}-\frac{e^{\phi / 2}}{4 !} \tilde{F}_{4}^{\mu_{1} \ldots \mu_{4}}\left[\star \mathbf{j}_{6} \wedge C_{1}\right]_{\mu_{1} \ldots \mu_{4}}^{\nu},
\end{aligned}
$$

where we have defined $\tilde{F}_{6}=\star \tilde{F}_{4}$ and $\tilde{F}_{8}=\star \tilde{F}_{2}$, while the current conservation equations (2.23) lead to

$$
\begin{aligned}
d \star \mathbf{J}_{1}-\star \mathbf{J}_{3} \wedge H_{3}-e^{\phi / 2} \star \tilde{F}_{4} \wedge \star \mathbf{j}_{6} & =0, \\
d \star \mathbf{J}_{3}-\star \mathbf{j}_{6} & \wedge F_{4}-H_{3} \wedge \star \mathcal{J}_{5}=0, \\
d \star \mathbf{j}_{2}=0, \quad d \star \mathbf{j}_{6}=0, \quad d \star \mathcal{J}_{5} & =H_{3} \wedge \star \mathcal{J}_{7}, \quad d \star \mathcal{J}_{7}=0 .
\end{aligned}
$$

Type IIB supergravity. We now consider restricting the equations of motion (2.18)(2.19) to $q=-1,1,3$. The resulting equations of motion can be obtained from the action ${ }^{22}$

$$
\begin{aligned}
I= & \frac{1}{16 \pi G} \int_{\mathcal{M}_{10}}\left[\star R-\frac{1}{2} d \phi \wedge \star d \phi-\frac{1}{2} e^{-\phi} H_{3} \wedge \star H_{3}-\frac{1}{2} \sum_{q=-1,1} e^{a_{q} \phi} \tilde{F}_{q+2} \wedge \star \tilde{F}_{q+2}\right] \\
& -\frac{1}{16 \pi G} \int_{\mathcal{M}_{10}}\left[\frac{1}{4} \tilde{F}_{5} \wedge \star \tilde{F}_{5}+\frac{1}{2} C_{3} \wedge H_{3} \wedge F_{4}\right]
\end{aligned}
$$

\footnotetext{
${ }^{21}$ We note that before coupling sources to the equations of motion, which can be obtained from (B.1), we use the Bianchi identities $d H_{3}=d \tilde{F}_{q+2}=0, q=0,2$ in vacuum in order to simplify them. This simplification is such that the equations of motion for type IIA are those which can be obtained from (2.18) by taking all current sources to be zero and restricting to $q=0,2,4,6$. We proceed similarly for type IIB.

${ }^{22}$ We are following the conventions of [44] but have redefined $C_{4} \rightarrow C_{4}-(1 / 2) B_{2} \wedge C_{2}$ for convenience.
} 
supplemented with the self-duality relation $\tilde{F}_{5}=\star \tilde{F}_{5}$. In the presence of sources, the equations of motion read

$$
\begin{array}{r}
d\left(e^{-\phi} \star H_{3}-e^{\phi} \star \tilde{F}_{3} \wedge C_{0}-\frac{1}{2} \star \tilde{F}_{5} \wedge C_{2}+\frac{1}{2} C_{4} \wedge F_{3}\right)=-16 \pi G \star \mathbf{j}_{2}, \\
d\left(e^{2 \phi} \star F_{1}\right)+e^{\phi} H_{3} \wedge \star \tilde{F}_{3}=-16 \pi G \star \mathbf{J}_{0}, \\
d\left(e^{\phi} \star \tilde{F}_{3}\right)+H_{3} \wedge \star \tilde{F}_{5}=-16 \pi G \star \mathbf{J}_{2}, \\
d\left(\star \tilde{F}_{5}\right)-H_{3} \wedge F_{3}=-16 \pi G \star \mathbf{J}_{4} .
\end{array}
$$

The equations of motion for the probe brane can be obtained by restricting (2.22) take the form

$$
\begin{aligned}
\nabla_{\mu} \mathbf{T}^{\mu \nu}= & \frac{1}{2 !} H_{3}^{\nu \mu_{1} \mu_{2}} \mathbf{j}_{2 \mu_{1} \mu_{2}}+\frac{e^{-\phi}}{6 !} H_{7}^{\nu \mu_{1} \ldots \mu_{6}} \mathbf{j}_{6 \mu_{1} \ldots \mu_{6}}+\mathbf{j}_{\phi} \partial^{\nu} \phi \\
& +F_{1}^{\nu} \mathbf{J}_{0}+\frac{1}{2 !}\left(\tilde{F}_{3}^{\nu \mu_{1} \ldots \mu_{2}}+H_{3}^{\nu \mu_{1} \mu_{2}} C_{0}\right) \mathbf{J}_{2 \mu_{1} \mu_{2}} \\
& +\frac{1}{4 !}\left(\tilde{F}_{5}^{\nu \mu_{1} \ldots \mu_{4}}+3 H_{3}^{\nu \mu_{1} \mu_{2}} C_{2}^{\mu_{3} \ldots \mu_{4}}\right) \mathbf{J}_{4 \mu_{1} \ldots \mu_{4}} \\
& +e^{\phi}\left(\frac{1}{6 !} \tilde{F}_{7}^{\nu \mu_{1} \ldots \mu_{6}}+\frac{1}{2 \cdot 4 !} H_{3}^{\nu \mu_{1} \mu_{2}} C_{4}^{\mu_{3} \ldots \mu_{6}}\right) \mathcal{J}_{6 \mu_{1} \ldots \mu_{6}} \\
& +\frac{e^{2 \phi}}{8 !} \tilde{F}_{9}^{\nu \mu_{1} \ldots \mu_{8}} \mathcal{J}_{8 \mu_{1} \ldots \mu_{8}}-\frac{e^{\phi}}{3 !} \tilde{F}_{3}^{\mu_{1} \ldots \mu_{3}}\left[\star \dot{j}_{6} \wedge C_{0}\right]_{\mu_{1} \ldots \mu_{3}}^{\nu},
\end{aligned}
$$

where we have defined $\tilde{F}_{7}=\star \tilde{F}_{3}$ and $\tilde{F}_{9}=\star \tilde{F}_{1}$, while the current conservation equations (2.23) lead to

$$
\begin{aligned}
& d \star \mathbf{J}_{2}+H_{3} \wedge \star \mathbf{J}_{4}+\star \mathbf{j}_{6} \wedge \tilde{F}_{5}=0, \\
& d \star J_{4}-\star \mathbf{j}_{6} \wedge F_{3}+\star \mathcal{J}_{6} \wedge \tilde{F}_{5}=0, \\
& d \star j_{2}=0, \quad d \star \mathfrak{j}_{6}=0, \quad d \star \mathcal{J}_{6}=H_{3} \wedge \star \mathcal{J}_{8}, \quad d \star \mathcal{J}_{8}=0 .
\end{aligned}
$$

Note that the magnetic force associated to $F_{5}$ in (B.7) was exchanged by a Lorentz type force as a consequence of the self-duality relation (2.21). Also note that there is no conservation equation associated with $\mathbf{J}_{0}$.

\section{Effective currents, charges and constraint equations}

In this appendix we provide the effective currents and charges for the several black brane solutions used in the main text.

\section{C.1 Black branes carrying $q=p$-brane charge}

The asymptotic stress-energy tensor and current of the charged black $p$-brane solution (3.1) take the form

$$
T_{a b}=\epsilon u_{a} u_{b}+P P_{a b}, \quad J_{p+1}=Q_{p}{ }_{(p+1)} \mathbf{1} .
$$


The stress-energy tensor is of the form of a perfect fluid with the energy density and (negative) pressure given by

$$
\epsilon=\frac{\Omega_{(n+1)}}{16 \pi G} r_{0}^{n}\left(n+1+n N \sinh ^{2} \alpha\right), \quad P=-\frac{\Omega_{(n+1)}}{16 \pi G} r_{0}^{n}\left(1+n N \sinh ^{2} \alpha\right),
$$

while the charge density and conjugate electric potential are

$$
Q_{p}=\frac{\Omega_{(n+1)}}{16 \pi G} n \sqrt{N} r_{0}^{n} \cosh \alpha \sinh \alpha, \quad \Phi_{p}=\sqrt{N} \tanh \alpha .
$$

Alternatively, we can express the stress-energy tensor as [20]

$$
T_{a b}=\mathcal{T} s\left(u_{a} u_{b}-\frac{1}{n} \gamma_{a b}\right)-\gamma_{a b} Q_{p} \Phi_{p}
$$

with the temperature and entropy density

$$
\mathcal{T}=\frac{n}{4 \pi r_{0}(\cosh \alpha)^{N}}, \quad s=\frac{\Omega_{(n+1)}}{4 G} r_{0}^{n+1}(\cosh \alpha)^{N} .
$$

\section{C.2 Black branes carrying Maxwell charge}

The asymptotic stress-energy tensor and current of the charged black $p$-brane given with Maxwell charge (4.15) take the form

$$
T_{a b}=\epsilon u_{a} u_{b}+P P_{a b}, \quad J_{1}^{a}=\mathcal{Q} u^{a},
$$

where the energy density and pressure are given by

$$
\epsilon=\frac{\Omega_{(n+1)}}{16 \pi G} r_{0}^{n}\left(n+1+n N \sinh ^{2} \alpha\right), \quad P=-\frac{\Omega_{(n+1)}}{16 \pi G} r_{0}^{n},
$$

while the charge density, chemical potential, temperature and entropy density are the same as in (C.3) and (C.5), respectively.

\section{C.3 D $p$-F1 bound state}

In this appendix we briefly review the effective currents and charges of the $\mathrm{D} p$-F1 bound state solution considered in section 3.2. For convenience we will take $\varphi=0$. The stressenergy tensor can be expressed in the form [18]

$$
T_{a b}=\epsilon u_{a} u_{b}+P v_{a} v_{b}+P_{\perp} \perp_{a b},
$$

where $\perp_{a b}=\eta_{a b}+u_{a} u_{b}-v_{a} v_{b}$ and the energy density and pressures are

$$
\begin{aligned}
\epsilon & =\frac{\Omega_{(n+1)}}{16 \pi G} r_{0}^{n}\left(n+1+n \sinh ^{2} \alpha\right), \\
P & =-\frac{\Omega_{(n+1)}}{16 \pi G}\left(1+n \sinh ^{2} \alpha\right), \quad P_{\perp}=-\frac{\Omega_{(n+1)}}{16 \pi G}\left(1+n \sinh ^{2} \alpha \cos ^{2} \xi\right) .
\end{aligned}
$$

Furthermore, we have the currents

$$
j_{2}=\mathcal{Q}_{\mathrm{F} 1} u \wedge v, \quad J_{p-1}=\mathcal{Q}_{p-2} \star_{(p+1)}(u \wedge v), \quad J_{p+1}=Q_{p} \star_{(p+1)} \mathbf{1},
$$


where the string and top charges are given by

$$
\mathcal{Q}_{\mathrm{F} 1}=\sin \xi \mathcal{Q}, \quad Q_{p}=\cos \xi \mathcal{Q}, \quad \text { with } \quad \mathcal{Q}=\frac{\Omega_{(n+1)}}{16 \pi G} n r_{0}^{n} \cosh \alpha \sinh \alpha,
$$

and electric potentials (conjugate to the charges) are

$$
\Phi_{\mathrm{F} 1}=\sin \xi \Phi, \quad \Phi_{p}=\cos \xi \Phi, \quad \text { with } \quad \Phi=\tanh \alpha .
$$

The charge associated with the $(p-1)$-current $J_{p-1}$ can be expressed in terms of the above

$$
\mathcal{Q}_{p-2}=\Phi_{p} \mathcal{Q}_{\mathrm{F} 1}=\Phi_{\mathrm{F} 1} Q_{p} .
$$

We note that for $\xi=0$, the effective currents and charges reduces to the one given in appendix C.1 (in ten dimensions where $N=1$ ). Introducing the worldvolume metric $\gamma_{a b}$ and the projector $h_{a b}=-u_{a} u_{b}+v_{a} v_{b}$, we can alternatively express the stress-energy tensor as [20]

$$
T_{a b}=\mathcal{T} s\left(u_{a} u_{b}-\frac{1}{n} \gamma_{a b}\right)-h_{a b} \mathcal{Q}_{\mathrm{F} 1} \Phi_{\mathrm{F} 1}-\gamma_{a b} Q_{p} \Phi_{p}
$$

with the temperature and entropy density

$$
\mathcal{T}=\frac{n}{4 \pi r_{0} \cosh \alpha}, \quad s=\frac{\Omega_{(n+1)}}{4 G} r_{0}^{n+1} \cosh \alpha .
$$

\section{C.4 D $p$-F1 constraint equations}

Let the components of the Einstein equations be denoted by $\mathcal{E}_{\mu \nu}=G_{\mu \nu}-8 \pi G T_{\mu \nu}$ and let the components of the l.h.s. of each of the eqs. (B.2) (eqs. (B.6)) for type IIA(B) be denoted by $\mathcal{M}_{X}^{\mu_{1} \mu_{2} \ldots}$ where $X$ denotes the associated field. Furthermore let the components of the Hodge dual of those equations be denoted by $\mathcal{N}_{X}^{\mu_{1} \mu_{2} \ldots}$. Then, the constraint equations (3.36) appear in the following linear combinations of the system equations

$$
\begin{aligned}
\nabla_{a} T^{a b}-\mathcal{F}^{b} & \propto \mathcal{E}_{r}{ }^{b}+c_{1} \mathcal{M}_{A_{p+1}}^{b \Omega}+c_{2} \mathcal{M}_{B}^{b \sigma_{2} \ldots \sigma_{p} \Omega}+c_{3} \mathcal{M}_{A_{p-1}}^{b \sigma_{0} \sigma_{1} \Omega} \\
\nabla_{a} j_{2}^{a b} & \propto \mathcal{N}_{B}^{r b}, \\
\nabla_{a} J_{p+1}^{a a_{1} \ldots a_{p}} & \propto \mathcal{N}_{A_{p+1}}^{r a_{1} \ldots a_{p}}, \\
\nabla_{a} J_{p-1}^{a a_{1} \ldots a_{p-2}}-\frac{1}{3 !} \mathcal{H}_{a b c} J_{p+1}^{a b c a_{1} \ldots a_{p-2}} & \propto \mathcal{N}_{A_{p+1}}^{r \sigma_{0} \sigma_{1} a_{1} \ldots a_{p-2}}+c_{4} \mathcal{N}_{A_{p-1}}^{r a_{1} \ldots a_{p-2}},
\end{aligned}
$$

where $c_{i}$ are $r$-dependent functions, $\Omega$ collectively denotes the coordinates on the transverse sphere and the F1-string is aligned along the $\sigma^{1}$-direction. $\mathcal{F}^{b}$ is the collection of force terms appearing in eq. (3.36). Notice that the combinations of the system equations are exactly such that the l.h.s. of eqs. (C.16) is $r$-independent.

\section{Entropy current analysis with a dilaton forcing function}

In this appendix we perform an entropy current analysis of the forced fluids considered in section 4.3 and show that in order to have stationary flows one must require the fluid to be aligned with a worldvolume Killing vector field. This analysis follows closely that of [72]. 
The fluids of section 4.3 have only the temperature $\mathcal{T}$ and the fluid velocity $u^{a}$ as degrees of freedom. These fluids are characterized also by a conserved total charge $Q_{p}$ but this charge is not a degree freedom, instead it only labels different families of such fluids. The thermodynamic properties given in appendix (C.1) are those of a neutral perfect fluid. Therefore, the dynamical equations along the worldvolume are just those of (4.37), which we write explicitly as

$$
u^{a} \partial_{a} \mathcal{T}=-\frac{1}{\mathcal{T}} \frac{\partial \mathcal{T}}{\partial s}\left(\mathcal{T} s \theta+j_{\phi} \dot{\varphi}\right), \quad P_{b}^{c} \partial^{b} \mathcal{T}=\frac{1}{s} P^{c}{ }_{b}\left(j_{\phi} \partial^{b} \varphi-\mathcal{T} s a^{b}\right)
$$

where we have defined $\dot{\varphi}=u^{a} \partial_{a} \varphi$ and introduced the fluid expansion $\theta$ via the decomposition

$$
\nabla_{a} u_{b}=-u_{a} a_{b}+\sigma_{a b}+\omega_{a b}+\frac{\theta}{p} P_{a b},
$$

where $\sigma_{a b}$ and $\omega_{a b}$ are the fluid shear and vorticity respectively. Up to first order in derivatives the most general stress-energy tensor, dilaton current and entropy current allowed by symmetries $\operatorname{are}^{23}$

$$
\begin{aligned}
T^{a b} & =P \gamma^{a b}+(\epsilon+P) u^{a} u^{b}-\zeta \sigma^{a b}-\eta \theta P^{a b}, \\
j_{\phi} & =j_{\phi}^{(0)}+\alpha_{1} \theta+\alpha_{2} \dot{\varphi}, \\
J_{s}^{a} & =s u^{a}+\beta_{1} \theta u^{a}+\beta_{2} a^{a}+\alpha_{3} u^{a} \dot{\varphi}+\alpha_{4} P^{a}{ }_{b} \partial^{b} \varphi,
\end{aligned}
$$

where all coefficients $\zeta, \eta, \beta_{i}, \alpha_{i}$ are functions of the temperature $\mathcal{T}$ and $j_{\phi}^{(0)}$ is the leading order dilaton current, which in the case of the branes of section 4.3 is given by $a_{p} Q_{p} \Phi_{p} / 2$, however, we have left it arbitrary in our analysis in this appendix. ${ }^{24}$

We wish to impose positivity of the divergence of the entropy current $\nabla_{a} J_{s}^{a} \geq 0$, thereby ensuring that the second law of thermodynamics is satisfied. Using (D.1) we find

$$
\begin{aligned}
\nabla_{a} J_{s}^{a}= & \zeta \sigma^{2}+\eta \theta^{2}+\left(\beta_{1}-s \beta_{1}^{\prime} \frac{\partial \mathcal{T}}{\partial s}+\frac{\beta_{2}}{p}\right) \theta^{2}-\beta_{2}^{\prime} a^{2}+\beta_{2}\left(\sigma^{2}+\omega^{2}\right) \\
& +\left(\beta_{1}+\beta_{2}\right) u^{a} \nabla_{a} \theta+\beta_{2} u^{a} u^{b} \mathcal{R}_{a b} \\
& -\frac{j_{\phi}^{(0)}}{\mathcal{T}} \dot{\varphi}-\frac{\alpha_{1}}{\mathcal{T}} \theta \dot{\varphi}-\frac{\alpha_{2}}{\mathcal{T}} \dot{\varphi}^{2} \\
& +\left(\alpha_{3}+\alpha_{4}-\frac{\partial \mathcal{T}}{\partial s} s \alpha_{3}^{\prime}-\frac{j_{\phi}^{(0)}}{\mathcal{T}} \frac{\partial \mathcal{T}}{\partial s} \beta_{1}^{\prime}\right) \theta \dot{\varphi}+\left(\alpha_{3}+\alpha_{4}-\alpha_{4}^{\prime} \mathcal{T}-\frac{j_{\phi}^{(0)}}{s} \beta_{2}^{\prime}\right) a^{b} \partial_{b} \varphi \\
& +\left(\frac{j_{\phi}^{(0)}}{s} \alpha_{4}^{\prime} P^{a b} \partial_{a} \varphi \partial_{b} \varphi+\alpha_{4} P^{a b} \partial_{a} \partial_{b} \varphi+\alpha_{3} u^{a} u^{b} \partial_{a} \partial_{b} \varphi\right)-\frac{j_{\phi}^{(0)}}{\mathcal{T}} \frac{\partial \mathcal{T}}{\partial s} \alpha_{3}^{\prime} \dot{\varphi}^{2},
\end{aligned}
$$

where we have defined $\sigma^{2}=\sigma_{a b} \sigma^{a b}$ and $\omega^{2}=\omega_{a b} \omega^{b a}$ and introduced the Ricci tensor $\mathcal{R}_{a b}$ on the worldvolume. The prime denotes derivatives with respect to $\mathcal{T}$. The first two lines

\footnotetext{
${ }^{23}$ One can also consider elastic corrections due to deformations of the surface where the fluid lives as in [72] but we are not concerned with these corrections here.

${ }^{24}$ In particular, the case studied in [10] has $j_{\phi}^{(0)}=0$.
} 
in (D.4) are those that are also obtained when no dilaton is present in the background. In particular the second line, being linear in fluid data requires $\beta_{1}=\beta_{2}=0$ and hence the first line requires the usual result $\zeta \geq 0, \eta \geq 0$ which is unaffected in the presence of the dilaton. The first three terms in the last line in (D.4) are linear in independent data and had been classified in [10]. Therefore, if we wish to require positivity of the divergence of the entropy current for arbitrary background source $\varphi$ we must set $\alpha_{3}=\alpha_{4}=0$. We are left with the third line in (D.4). The first term in the third line is linear in the fluid data but $j_{\phi}^{(0)}$ is non-zero. Therefore one must require $-j_{\phi}^{(0)} \dot{\varphi} \geq 0 .{ }^{25}$ If $j_{\phi}^{(0)}$ is constant, for example, this condition will impose restrictions on the driving force $\dot{\varphi}$. The second term in the third line is linear in fluid data and therefore, for arbitrary background sources we must have that $\alpha_{1}=0$. The third term in the third line is quadratic in the fluid data and therefore we obtain the condition $\alpha_{2} \leq 0$.

Consider the forced fluid dynamics case analyzed in [10]. To first order in derivatives the dilaton current found there is given by

$$
j_{\phi}=-\frac{1}{16 \pi G}(\pi \mathcal{T})^{3} \dot{\varphi},
$$

and hence we identify $j_{\phi}^{(0)}=0$ and $\alpha_{1}=0, \alpha_{2}=-(\pi \mathcal{T})^{3} /(16 \pi G)<0$ in agreement with the analysis above. The entropy current obtained in [10] contains no first order corrections, also in agreement with the analysis presented here.

Consider now the stationary case for which there is no entropy production $\nabla_{a} J_{s}^{a}=0$. Due to the presence of non-zero viscosities $\zeta, \eta$ and leading order dilaton current $j_{\phi}^{(0)}$, stationary configurations must satisfy $\theta=\sigma^{a b}=\dot{\varphi}=0 .{ }^{26}$ Indeed, this is only possible if the fluid velocity is aligned with a worldvolume Killing vector field, i.e., $u^{a}=\mathbf{k}^{a} / \mathbf{k}$.

Open Access. This article is distributed under the terms of the Creative Commons Attribution License (CC-BY 4.0), which permits any use, distribution and reproduction in any medium, provided the original author(s) and source are credited.

\section{References}

[1] G. Policastro, D.T. Son and A.O. Starinets, The Shear viscosity of strongly coupled $N=4$ supersymmetric Yang-Mills plasma, Phys. Rev. Lett. 87 (2001) 081601 [hep-th/0104066] [INSPIRE].

[2] P. Kovtun, D.T. Son and A.O. Starinets, Viscosity in strongly interacting quantum field theories from black hole physics, Phys. Rev. Lett. 94 (2005) 111601 [hep-th/0405231] [INSPIRE].

[3] S. Bhattacharyya, V.E. Hubeny, S. Minwalla and M. Rangamani, Nonlinear Fluid Dynamics from Gravity, JHEP 02 (2008) 045 [arXiv:0712.2456] [INSPIRE].

[4] R. Baier, P. Romatschke, D.T. Son, A.O. Starinets and M.A. Stephanov, Relativistic viscous hydrodynamics, conformal invariance and holography, JHEP 04 (2008) 100 [arXiv:0712.2451] [INSPIRE].

\footnotetext{
${ }^{25}$ This specific condition had already been noticed in [10] using other arguments.

${ }^{26}$ We are assuming here that the transport coefficients are non-zero, which is indeed the case for the fluids we are considering $[9,12]$.
} 
[5] M. Rangamani, Gravity and Hydrodynamics: Lectures on the fluid-gravity correspondence, Class. Quant. Grav. 26 (2009) 224003 [arXiv:0905.4352] [INSPIRE].

[6] R. Emparan, T. Harmark, V. Niarchos and N.A. Obers, World-Volume Effective Theory for Higher-Dimensional Black Holes, Phys. Rev. Lett. 102 (2009) 191301 [arXiv:0902.0427] [INSPIRE].

[7] R. Emparan, T. Harmark, V. Niarchos and N.A. Obers, Essentials of Blackfold Dynamics, JHEP 03 (2010) 063 [arXiv:0910.1601] [INSPIRE].

[8] J. Camps, R. Emparan and N. Haddad, Black Brane Viscosity and the Gregory-Laflamme Instability, JHEP 05 (2010) 042 [arXiv: 1003.3636] [INSPIRE].

[9] R. Emparan, V.E. Hubeny and M. Rangamani, Effective hydrodynamics of black D3-branes, JHEP 06 (2013) 035 [arXiv: 1303.3563] [INSPIRE].

[10] S. Bhattacharyya, R. Loganayagam, S. Minwalla, S. Nampuri, S.P. Trivedi and S.R. Wadia, Forced Fluid Dynamics from Gravity, JHEP 02 (2009) 018 [arXiv:0806.0006] [INSPIRE].

[11] J. Gath and A.V. Pedersen, Viscous asymptotically flat Reissner-Nordström black branes, JHEP 03 (2014) 059 [arXiv: 1302.5480] [INSPIRE].

[12] A. Di Dato, J. Gath and A.V. Pedersen, Probing the Hydrodynamic Limit of (Super)gravity, JHEP 04 (2015) 171 [arXiv: 1501.05441] [INSPIRE].

[13] R. Emparan, T. Harmark, V. Niarchos, N.A. Obers and M.J. Rodriguez, The Phase Structure of Higher-Dimensional Black Rings and Black Holes, JHEP 10 (2007) 110 [arXiv: 0708.2181] [INSPIRE].

[14] M.M. Caldarelli, R. Emparan and M.J. Rodriguez, Black Rings in (Anti)-deSitter space, JHEP 11 (2008) 011 [arXiv:0806.1954] [INSPIRE].

[15] J. Camps, R. Emparan, P. Figueras, S. Giusto and A. Saxena, Black Rings in Taub-NUT and D0-D6 interactions, JHEP 02 (2009) 021 [arXiv:0811.2088] [INSPIRE].

[16] R. Emparan, T. Harmark, V. Niarchos and N.A. Obers, New Horizons for Black Holes and Branes, JHEP 04 (2010) 046 [arXiv:0912.2352] [INSPIRE].

[17] G. Grignani, T. Harmark, A. Marini, N.A. Obers and M. Orselli, Heating up the BIon, JHEP 06 (2011) 058 [arXiv: 1012.1494] [INSPIRE].

[18] M.M. Caldarelli, R. Emparan and B. Van Pol, Higher-dimensional Rotating Charged Black Holes, JHEP 04 (2011) 013 [arXiv: 1012.4517] [INSPIRE].

[19] J. Armas and N.A. Obers, Blackfolds in (Anti)-de Sitter Backgrounds, Phys. Rev. D 83 (2011) 084039 [arXiv: 1012.5081] [inSPIRE].

[20] R. Emparan, T. Harmark, V. Niarchos and N.A. Obers, Blackfolds in Supergravity and String Theory, JHEP 08 (2011) 154 [arXiv:1106.4428] [INSPIRE].

[21] J. Armas, J. Camps, T. Harmark and N.A. Obers, The Young Modulus of Black Strings and the Fine Structure of Blackfolds, JHEP 02 (2012) 110 [arXiv:1110.4835] [INSPIRE].

[22] J. Camps and R. Emparan, Derivation of the blackfold effective theory, JHEP 03 (2012) 038 [Erratum ibid. 1206 (2012) 155] [arXiv:1201.3506] [INSPIRE].

[23] V. Niarchos and K. Siampos, M2-M5 blackfold funnels, JHEP 06 (2012) 175 [arXiv: 1205.1535] [INSPIRE]. 
[24] J. Armas, J. Gath and N.A. Obers, Black Branes as Piezoelectrics, Phys. Rev. Lett. 109 (2012) 241101 [arXiv: 1209.2127] [INSPIRE].

[25] J. Armas, J. Gath and N.A. Obers, Electroelasticity of Charged Black Branes, JHEP 10 (2013) 035 [arXiv: 1307.0504] [INSPIRE].

[26] J. Armas and M. Blau, Blackfolds, Plane Waves and Minimal Surfaces, JHEP 07 (2015) 156 [arXiv: 1503.08834] [INSPIRE].

[27] J. Armas and M. Blau, New Geometries for Black Hole Horizons, JHEP 07 (2015) 048 [arXiv: 1504.01393] [INSPIRE].

[28] J. Armas, T. Harmark, N.A. Obers, M. Orselli and A.V. Pedersen, Thermal Giant Gravitons, JHEP 11 (2012) 123 [arXiv: 1207.2789] [INSPIRE].

[29] J. Armas, N.A. Obers and A.V. Pedersen, Null-Wave Giant Gravitons from Thermal Spinning Brane Probes, JHEP 10 (2013) 109 [arXiv:1306.2633] [INSPIRE].

[30] V. Niarchos, Open/closed string duality and relativistic fluids, Phys. Rev. D 94 (2016) 026009 [arXiv: 1510.03438] [INSPIRE].

[31] G. Grignani, T. Harmark, A. Marini and M. Orselli, Born-Infeld/gravity correspondence, Phys. Rev. D 94 (2016) 066009 [arXiv:1602.01640] [INSPIRE].

[32] G. Grignani, T. Harmark, A. Marini, N.A. Obers and M. Orselli, Thermodynamics of the hot BIon, Nucl. Phys. B 851 (2011) 462 [arXiv:1101.1297] [INSPIRE].

[33] G. Grignani, T. Harmark, A. Marini and M. Orselli, Thermal DBI action for the D3-brane at weak and strong coupling, JHEP 03 (2014) 114 [arXiv:1311.3834] [INSPIRE].

[34] P. Pasti, D.P. Sorokin and M. Tonin, Covariant action for a $D=11$ five-brane with the chiral field, Phys. Lett. B 398 (1997) 41 [hep-th/9701037] [INSPIRE].

[35] V. Niarchos, Supersymmetric Perturbations of the M5 brane, JHEP 05 (2014) 023 [arXiv: 1402.4132] [INSPIRE].

[36] R.C. Myers and M.J. Perry, Black Holes in Higher Dimensional Space-Times, Annals Phys. 172 (1986) 304 [INSPIRE].

[37] T. Harmark and N.A. Obers, Black holes on cylinders, JHEP 05 (2002) 032 [hep-th/0204047] [INSPIRE].

[38] B. Kol, E. Sorkin and T. Piran, Caged black holes: Black holes in compactified space-times.

1. Theory, Phys. Rev. D 69 (2004) 064031 [hep-th/0309190] [InSPIRE].

[39] W. Rindler, Relativity: Special, General, and Cosmological, Oxford University Press, (2001).

[40] J. Bhattacharya, S. Bhattacharyya and S. Minwalla, Dissipative Superfluid dynamics from gravity, JHEP 04 (2011) 125 [arXiv:1101.3332] [INSPIRE].

[41] E. Bergshoeff, R. Kallosh, T. Ortín, D. Roest and A. Van Proeyen, New formulations of $D=10$ supersymmetry and D8 - O8 domain walls, Class. Quant. Grav. 18 (2001) 3359 [hep-th/0103233] [INSPIRE].

[42] G. Dall'Agata, K. Lechner and M. Tonin, $D=10, N=I I B$ supergravity., JHEP 07 (1998) 017 [hep-th/9806140] [INSPIRE].

[43] I.A. Bandos, A.J. Nurmagambetov and D.P. Sorokin, Various faces of type IIA supergravity, Nucl. Phys. B 676 (2004) 189 [hep-th/0307153] [INSPIRE]. 
[44] K. Becker, M. Becker and J. Schwarz, String Theory and M-Theory: A Modern Introduction, Cambridge University Press, (2006).

[45] I.A. Bandos, N. Berkovits and D.P. Sorokin, Duality symmetric eleven-dimensional supergravity and its coupling to M-branes, Nucl. Phys. B 522 (1998) 214 [hep-th/9711055] [INSPIRE].

[46] G.T. Horowitz and A. Strominger, Black strings and P-branes, Nucl. Phys. B 360 (1991) 197 [INSPIRE].

[47] J. Erdmenger, M. Haack, M. Kaminski and A. Yarom, Fluid dynamics of R-charged black holes, JHEP 01 (2009) 055 [arXiv:0809. 2488] [INSPIRE].

[48] N. Banerjee, J. Bhattacharya, S. Bhattacharyya, S. Dutta, R. Loganayagam and P. Surowka, Hydrodynamics from charged black branes, JHEP 01 (2011) 094 [arXiv:0809.2596] [INSPIRE].

[49] D. Gorbonos and B. Kol, A dialogue of multipoles: Matched asymptotic expansion for caged black holes, JHEP 06 (2004) 053 [hep-th/0406002] [INSPIRE].

[50] T. Harmark, Supergravity and space-time noncommutative open string theory, JHEP 07 (2000) 043 [hep-th/0006023] [INSPIRE].

[51] M. Crossley, P. Glorioso and H. Liu, Effective field theory of dissipative fluids, arXiv: 1511.03646 [INSPIRE].

[52] F.M. Haehl, R. Loganayagam and M. Rangamani, Topological $\sigma$-models $\mathcal{E} 3$ dissipative hydrodynamics, JHEP 04 (2016) 039 [arXiv:1511.07809] [INSPIRE].

[53] G.W. Gibbons and S.W. Hawking, Action Integrals and Partition Functions in Quantum Gravity, Phys. Rev. D 15 (1977) 2752 [InSPIRE].

[54] J. Armas, How Fluids Bend: the Elastic Expansion for Higher-Dimensional Black Holes, JHEP 09 (2013) 073 [arXiv: 1304.7773] [INSPIRE].

[55] G. Grignani, T. Harmark, A. Marini, N.A. Obers and M. Orselli, Thermal string probes in AdS and finite temperature Wilson loops, JHEP 06 (2012) 144 [arXiv:1201.4862] [INSPIRE].

[56] V. Niarchos and K. Siampos, Entropy of the self-dual string soliton, JHEP 07 (2012) 134 [arXiv: 1206.2935] [INSPIRE].

[57] V. Niarchos and K. Siampos, The black M2-M5 ring intersection spins, PoS (Corfu2012) 088 [arXiv: 1302.0854] [INSPIRE].

[58] J. Armas and M. Blau, Black probes of Schrödinger spacetimes, JHEP 08 (2014) 140 [arXiv: 1405.1301] [INSPIRE].

[59] D. Giataganas and K. Goldstein, Tension of Confining Strings at Low Temperature, JHEP 02 (2015) 123 [arXiv: 1411.4995] [INSPIRE].

[60] I. Bena, M. Graña and N. Halmagyi, On the Existence of Meta-stable Vacua in Klebanov-Strassler, JHEP 09 (2010) 087 [arXiv: 0912.3519] [INSPIRE].

[61] J. Blaback, U.H. Danielsson, D. Junghans, T. Van Riet, T. Wrase and M. Zagermann, (Anti-)Brane backreaction beyond perturbation theory, JHEP 02 (2012) 025 [arXiv: 1111.2605] [INSPIRE].

[62] I. Bena, J. Blåbäck and D. Turton, Loop corrections to the antibrane potential, JHEP 07 (2016) 132 [arXiv: 1602.05959] [INSPIRE]. 
[63] I.R. Klebanov and M.J. Strassler, Supergravity and a confining gauge theory: Duality cascades and chi SB resolution of naked singularities, JHEP 08 (2000) 052 [hep-th/0007191] [INSPIRE].

[64] S. Kachru, J. Pearson and H.L. Verlinde, Brane/flux annihilation and the string dual of a nonsupersymmetric field theory, JHEP 06 (2002) 021 [hep-th/0112197] [INSPIRE].

[65] I. Bena, G. Giecold, M. Graña, N. Halmagyi and S. Massai, The backreaction of anti-D3 branes on the Klebanov-Strassler geometry, JHEP 06 (2013) 060 [arXiv:1106.6165] [INSPIRE].

[66] I.R. Klebanov and S.S. Pufu, M-Branes and Metastable States, JHEP 08 (2011) 035 [arXiv: 1006.3587] [INSPIRE].

[67] I. Bena, G. Giecold and N. Halmagyi, The Backreaction of Anti-M2 Branes on a Warped Stenzel Space, JHEP 04 (2011) 120 [arXiv:1011.2195] [INSPIRE].

[68] S. Massai, Metastable Vacua and the Backreacted Stenzel Geometry, JHEP 06 (2012) 059 [arXiv:1110.2513] [INSPIRE].

[69] M. Gutperle and A. Strominger, Space-like branes, JHEP 04 (2002) 018 [hep-th/0202210] [INSPIRE].

[70] A. Sen, Rolling tachyon, JHEP 04 (2002) 048 [hep-th/0203211] [INSPIRE].

[71] D. Kutasov and V. Niarchos, Tachyon effective actions in open string theory, Nucl. Phys. B 666 (2003) 56 [hep-th/0304045] [INSPIRE].

[72] J. Armas, (Non)-Dissipative Hydrodynamics on Embedded Surfaces, JHEP 09 (2014) 047 [arXiv: 1312.0597] [INSPIRE].

[73] J. Armas and T. Harmark, Constraints on the effective fluid theory of stationary branes, JHEP 10 (2014) 063 [arXiv: 1406.7813] [INSPIRE].

[74] J. Armas, J. Bhattacharya and N. Kundu, Surface transport in plasma-balls, JHEP 06 (2016) 015 [arXiv: 1512.08514] [INSPIRE].

[75] J. Armas, N.A. Obers and M. Sanchioni, Gravitational Tension, Spacetime Pressure and Black Hole Volume, JHEP 09 (2016) 124 [arXiv: 1512.09106] [INSPIRE]. 